\title{
ON THE PRIMITIVE IRREDUCIBLE REPRESENTATIONS OF FINITELY GENERATED LINEAR GROUPS OF FINITE RANK
}

\author{
A. V. TUSHEV \\ May 3, 2021 \\ Department of Mathematics, Dnipro National University, \\ Gagarin Avenue 72, Dnipro, 49065, Ukraine \\ tushev@mmf.dnu.edu.ua
}

\begin{abstract}
In the paper we study finitely generated linear groups of finite rank which have faithful irreducible primitive representations over a field of characteristic zero. We prove that if an infinite finitely generated linear group $G$ of finite rank has a faithful irreducible primitive representation over a field of characteristic zero then the $F C$-center $\Delta(G)$ of $G$ is infinite.
\end{abstract}

\section{Introduction}

A group $G$ is said to have finite (Prufer) rank if there is a positive integer $m$ such that any finitely generated subgroup of $G$ may be generated by $m$ elements; the smallest $m$ with this property is the $\operatorname{rank} r(G)$ of $G$. A group $G$ is said to be of finite torsion-free rank if it has a finite series each of whose factor is either infinite cyclic or locally finite; the number $r_{0}(G)$ of infinite cyclic factors in such a series is the torsion-free rank of $G$.

If the group $G$ has a finite series each of whose factor is either cyclic or quasi-cyclic then $G$ is said to be minimax; the number $m(G)$ of infinite factors 
in such a series is the minimax length of $G$. If in such a series all infinite factors are cyclic then the group $G$ is said to be polycyclic; the number $h(G)$ of infinite factors in such a series is the Hirsch number of $G$. It is well known that $r_{0}(G)=m(G)=h(G)$ if the group $G$ is polycyclic.

Let $G$ be a group, $R$ be a ring and $R G$ be the group ring. Let $H$ be a subgroup of the group $G$ and let $U$ be a right $R H$-module. Since the group ring $R G$ can be considered as a left $R H$-module, we can define the tensor product $U \otimes_{R H} R G$, which is a right $R G$-module named the $R G$-module induced from the $R H$-module $U$. Moreover, if $M$ is an $R G$-module and $U \leq M$, then

$$
M=U \otimes_{R H} R G
$$

if and only if

$$
M=\oplus_{t \in T} U t,
$$

where $T$ - is a right transversal to subgroup $H$ in $G$.

If a $k G$-module $M$ of some representation $\varphi$ of a group $G$ over a field $k$ is induced from some $k H$-module $U$, where $H$ is a subgroup of the group $G$, then we say that the representation $\varphi$ is induced from a representation $\phi$ of subgroup $H$, where $U$ is the module of the representation $\phi$.

Eq. (21) shows that properties of the $R G$-module $M$ and the $R H$-module $U$ are closely related. So, Eq. (11) may be very useful if properties of the $R H$-module $U$ are well studied. For instance, in the case where the group $H$ is polycyclic, we have a deeply developed theory (see [25]). Eq. (1) also may be applied in the case where the group $G$ has finite torsion-free rank $r_{0}(G)$ if $r_{0}(H)<r_{0}(G)$ because then we can use the induction on $r_{0}(G)$. However, on the place of $r_{0}(G)$ there may be another rank of the group $G$ or the minimax length $m(G)$ if $G$ is minimax.

The subgroup $H \leq G$ which provides (11) is said to be a control subgroup for the $R G$-module $M$. Various types of control subgroups for modules over group rings of groups of finite rank were considered in [15 18].

Let $G$ be a group, let $k$ be a field and let $M$ be a $k G$-module. The module $M$ is said to be primitive if it is not induced from any $k H$-submodule for any subgroup $H<G$. Recall that the representation $\varphi$ is said to be faithful if $\operatorname{Ker} \varphi=1$, it is equal to $C_{G}(M)=1$.

Certainly, primitive irreducible modules are basic subjects for investigations when we are dealing with induced modules. Naturally, the following question appears: what can be said on the construction of a group $G$ if it has a faithful primitive irreducible representation over a field $k$ ? It should be 
noted that there are many results which show that the existence of a faithful irreducible representation of a group $G$ over a field $k$ may have essential influence on the structure of the group $G$ (see for instance [11 14, 18, 20]).

In [5] Harper solved a problem raised by Zaleskii and proved that any not abelian-by-finite finitely generated nilpotent group has an irreducible primitive representation over a not locally finite field. In [19] we proved that if a minimax nilpotent group $G$ of class 2 has a faithful irreducible primitive representation over a finitely generated field of characteristic zero then the group $G$ is finitely generated. In [6] Harper studied polycyclic groups which have faithful irreducible primitive representations. It is well known that any polycyclic group is finitely generated soluble of finite rank and meets the maximal condition for subgroups (in particular, for normal subgroups). In [18] we showed that in the class of soluble groups of finite rank with the maximal condition for normal subgroups only polycyclic groups may have faithful irreducible primitive representations over a field of characteristic zero.

If $G$ is a group then the $F C$-center $\Delta(G)=\left\{g \in G|| G: C_{G}(g) \mid<\infty\right\}$ of $G$ is a characteristic subgroup of $G$. In [6, Theorem A] Harper proved that if a polycyclic group $G$ has a faithful primitive irreducible representation over a field $k$ then $\Delta(G)$ is rather large in the sense that $\Delta(G) \cap H>1$ for any subgroup $1 \neq H$ of $G$ such that $\left|G: N_{G}(H)\right|<\infty$.

By Auslander theorem, any polycyclic group $G$ is linear and it is well known that the group $G$ is finitely generated of finite rank. In the presented paper we study finitely generated linear (over a field of characteristic zero) groups of finite rank which have faithful irreducible primitive representations over a field of characteristic zero. We prove that if an infinite finitely generated linear group $G$ of finite rank has a faithful irreducible primitive representation over a field of characteristic zero then $\Delta(G)$ is infinite (see Theorem 6.1).

Our methods of investigations are based on the following techniques introduced by Brookes in [4] for the case of polycyclic groups. Let $N$ be a group and let $K$ be a normal subgroup of $N$ such that the quotient group $N / K$ is torsion-free abelian of finite rank. Let $R$ be a ring and let $W$ be a finitely generated $R N$-module. Let $I$ be an $N$-invariant ideal of $R K$ such that $\left|K / I^{\dagger}\right|<\infty$ and $k=R /(R \cap I)$ is a field, where $I^{\dagger}=G \cap(1+I)$. Then $N / I^{\dagger}$ has a central subgroup $A$ of finite index (see Lemma 2.2(i)). So, the quotient module $W / W I$ may be considered as a finitely generated $k A$-module. One may ask how deeply properties of the $R N$-module $W$ are related with properties of the $k A$-module $W / W I$. However, for our needs 
the approach is quite fruitful and we consider relations between $W$ and a set of prime ideals of $k A$ which are minimal over $A n n_{k A}(W / W I)$.

\section{Auxiliary Results}

Let $A, B$ and $H$ be subgroups of a group $G$ such that $B$ is a normal subgroup of $A$. We say that $A$ is an $H$-invariant subgroup if $H \leqslant N_{G}(A)$. A section $A / B$ will be called an $H$-invariant section of $G$ if $A$ and $B$ are $H$-invariant subgroups.

Let $H$ be a subgroup of a group $G$, the subgroup $H$ is said to be dense in $G$ if for any $g \in G$ there is an integer $n \in \mathbb{N}$ such that $g^{n} \in H$. If $g^{n} \in G \backslash H$ for any $n \in \mathbb{N}$ and any $g \in G \backslash H$ then the subgroup $H$ is said to be isolated in $G$. If the group $G$ is locally nilpotent then the isolator $i s_{G}(H)=\left\{g \in G \mid g^{n} \in H\right.$ for some $\left.n \in \mathbb{N}\right\}$ of $H$ in $G$ is a subgroup of $G$ and if $H$ is a normal subgroup then so is $i s_{G}(H)$.

If $G$ is a group then $G^{\prime}$ denotes the derived subgroup of $G$.

Lemma 2.1. Let $D$ be a nilpotent torsion-free normal subgroup of nilpotency class two of a group $G$. Let $K$ be the centre of $D$ and let $L=i s_{D}\left(D^{\prime}\right)$. Suppose that there is a G-invariant subgroup $P \leqslant D$ such that $L \leqslant P$, the quotient group $P / L$ is polycyclic, $P \cap K=L$ and $P K$ has finite index in $D$. Then:

(i) $P^{\prime}$ is a $G$-invariant polycyclic dense subgroup of $L$;

(ii) for any finitely generated subgroup $H \leqslant G$ the group $D$ has an $H$ invariant normal polycyclic non-abelian subgroup.

Proof. It is well known that in any group $D$ of nilpotency class two the following relation holds for any $a, b, c, d \in D$ :

$$
[a b, c d]=[a, c][a, d][b, c][b, d] .
$$

(i) It follows from (3) that $\left[a^{m}, b^{n}\right]=[a, b]^{m n}$ for any $a, b \in D$ and any $m, n \in \mathbb{N}$. Then, as $P K$ has finite index in $D$, it implies that $(P K)^{\prime}$ is a dense subgroup of $D^{\prime}$ and hence $(P K)^{\prime}$ is a dense subgroup of $L=i s_{D}\left(D^{\prime}\right)$. Since $K$ is the centre of $D$, it follows from (3) that $(P K)^{\prime}=P^{\prime}$ and hence $P^{\prime}$ is a dense subgroup of $L$. 
As the quotient group $P / L$ is finitely generated, there is a finitely generated subgroup $A=\left\langle a_{1}, \ldots, a_{t}\right\rangle \leqslant P$ such that $P=L A$. Then it easily follows from (3) that $P^{\prime}=(L A)^{\prime}=A^{\prime}=\left(\left\langle a_{1}, \ldots, a_{t}\right\rangle\right)^{\prime}=\left\langle\left[a_{i}, a_{j}\right] \mid 1 \leqslant i, j \leqslant t\right\rangle$ and hence $P^{\prime}$ is a finitely generated subgroup of $P$. Since $P$ is a $G$-invariant subgroup and $[a, b]^{g}=\left[a^{g}, b^{g}\right]$ for all $a, b \in D$ and $g \in G$, we can conclude that $P^{\prime}$ is a $G$-invariant subgroup.

(ii) At first, we should note that $L$ is a $G$-invariant subgroup as the isolator in $P$ of the $G$-invariant subgroup $P^{\prime}$. Since $P^{\prime}$ is a finitely generated dense subgroup of an abelian torsion-free subgroup $L$, we can conclude that $r(L)<\infty$ and hence $L / P^{\prime}$ is an union of an ascending series of its finite $G$-invariant subgroups.

As the quotient group $P / L$ is finitely generated, there is a finitely generated subgroup $A=\left\langle a_{1}, \ldots, a_{t}\right\rangle \leqslant P$ such that $P=L A$. Let $H=\left\langle h_{1}, \ldots, h_{m}\right\rangle$ be a finitely generated subgroup of $G$. As $P=L A$, for any $a_{i}$ and $h_{j}$ there are $u_{i j} \in L$ and $v_{i j} \in A$ such that $a_{i}{ }^{h_{j}}=u_{i j} v_{i j}$, where $1 \leqslant i \leqslant t$ and $1 \leqslant j \leqslant m$. Since $L / P^{\prime}$ is an union of an ascending series of its finite $G$-invariant subgroups, there is a $G$-invariant subgroup $T \leqslant L$ such that $P^{\prime} \leqslant T,\left|T / P^{\prime}\right|<\infty$ and all $u_{i j} \in T$ and therefore, as $a_{i}^{h_{j}}=u_{i j} v_{i j}$, we see that all $a_{i}{ }^{h_{j}} \in T A$, where $1 \leqslant i \leqslant t$ and $1 \leqslant j \leqslant m$. It implies that $T A$ is an $H$-invariant normal polycyclic non-abelian subgroup of $D$.

Lemma 2.2. Let $G$ be a group of finite rank. Then:

(i) if the group $G$ has a finite subgroup $D$ such that the quotient group $G / D$ is torsion-free abelian then $G$ has a characteristic central torsion-free subgroup A of finite index;

(ii) if the group $G$ is finitely generated linear and has no polycyclic subgroups of finite index then $G$ has a finite series $L \leqslant G_{0} \leqslant G$ of normal subgroups such that $\left|G / G_{0}\right|<\infty$, the quotient group $G_{0} / L$ is polycyclic, the subgroup $L$ is torsion-free nilpotent minimax and has no non-abelian torsion-free polycyclic G-invariant sections.

Proof. (i) As $G^{\prime}$ is a characteristic subgroup of $G$, it is easy to note that $C=$ $C_{G}\left(G^{\prime}\right)$ is a characteristic subgroup of $G$. Then $B=C^{m}$ is a characteristic subgroup of $G$, where $m=|D|$. Since $\left|G^{\prime}\right|<\infty$ and $C=C_{G}\left(G^{\prime}\right)$, we can conclude that $|G: C|<\infty$. As the group $G$ has finite rank, it is easy to note that $\left|C: C^{m}\right|<\infty$ and hence we can conclude that $|G: B|<\infty$. For any $a, b \in C$ and any $g \in G$ we have $[a b, g]=[a, g]^{b}[b, g]$ and, as $[a, g] \in G^{\prime}$ and 
$b \in C=C_{G}\left(G^{\prime}\right)$, we can conclude that

$$
[a b, g]=[a, g][b, g] .
$$

Any element $a \in B=C^{m}$ may be presented in the form $a=a_{1}{ }^{m} \ldots a_{t}{ }^{m}$, where $a_{i} \in C$ and $1 \leqslant i \leqslant t$. It follows from (4) that for any $g \in G$ we have $[a, g]=\left[a_{1}, g\right]^{m} \ldots\left[a_{t}, g\right]^{m}$ then, as $m=|D|$ and $\left[a_{i}, g\right] \in G^{\prime} \leqslant D$, where $1 \leqslant i \leqslant t$, we can conclude that $[a, g]=1$. It implies that $B$ is a characteristic central subgroup of finite index in $G$. Since the quotient group $G / D$ is torsion-free, we see that so is $B /(B \cap D)$ and it easily implies that $A=B^{m}$ is a torsion-free characteristic central subgroup of finite index in $G$.

(ii) Since the group $G$ is linear over a field of characteristic zero and $r(G)<\infty$, it follows from Titts theorem [24, Theorem 10.17] that the group $G$ is soluble-by-finite. Then it follows from Kolchin-Maltsev theorem [24, Theorem 3.6] that the group $G$ has a finite series of normal subgroups $G_{1} \leqslant$ $G_{0} \leqslant G$ such that $\left|G / G_{0}\right|<\infty$, the quotient group $G_{0} / G_{1}$ is abelian and the subgroup $G_{1}$ is torsion-free nilpotent. As the group $G$ is finitely generated, by [10, Proposition 1.6.11], so is $G_{0}$ and hence the quotient group $G / G_{1}$ is polycyclic-by-finite. By [8, Proposition 5.2.8], the group $G_{0}$ is minimax. As $r(G)<\infty, G_{1}$ has a $G$-invariant subgroup $L$ such that the quotient group $G_{1} / L$ is infinite polycyclic of maximal possible torsion-free rank. It easily implies that $L$ has no $G$-invariant subgroup $Y$ such that the quotient group $N / Y$ is infinite polycyclic and, evidently, the quotient group $G_{0} / L$ is polycyclic.

Suppose that $L$ has a non-abelian torsion-free polycyclic $G$-invariant sections $A / B$. The section $A / B$ is acted by $G$ via conjugations and hence $L / C_{L}(A / B) \leqslant A u t(A / B)$, where $C_{L}(A / B)$ is a $G$-invariant subgroup of $L$. Then it follows from [7, Theorem 21.3.2, Theorem 21.2.1] that the quotient group $L / C_{L}(A / B)$ is polycyclic. Evidently, $C_{A}(A / B)=C_{L}(A / B) \cap A=C$, where $C / B$ is the centre of $A / B$. As the centre $C / B$ of the torsion-free nilpotent non-abelian group $A / B$ is isolated, we can conclude that $|A / C|=\infty$ and it easily implies that $\left|L / C_{L}(A / B)\right|=\infty$. But it is impossible, because $L$ has no $G$-invariant subgroup $Y$ such that the quotient group $L / Y$ is infinite polycyclic. Thus, we can conclude that $L$ has no non-abelian torsion-free polycyclic $G$-invariant sections.

Let $A$ be an abelian additive torsion-free group acted by a group $G$. Let $\bar{A}=A \otimes_{\mathbb{Z}} \mathbb{Q}$, we denote by $\operatorname{Soc}_{G} \bar{A}$ the socle of the $\mathbb{Q} G$-module $\bar{A}$ and put $\operatorname{Soc}_{G} A=\operatorname{Soc}_{G} \bar{A} \cap A$. It is not difficult to show that $\operatorname{Soc}_{G} A$ is an isolated 
$G$-invariant subgroup of $A$. We denote by $\Delta_{G}(A)$ the set of elements of $A$ which have finite orbits under the action of $G$, then $\Delta_{G}(A)$ is a $G$-invariant isolated subgroup of $A$.

Lemma 2.3. Let $A$ be an abelian torsion-free group acted by a group $G$ and let $H$ be a normal subgroup of finite index in $G$. Then:

(i) $\operatorname{Soc}_{H} A$ is an isolated $G$-invariant subgroup of $A$ such that $\Delta_{G}(A) \leqslant$ $\operatorname{Soc}_{H} A$ and $\Delta_{G}\left(\operatorname{Soc}_{H} A / \Delta_{G}(A)\right)$ is trivial;

(ii) $C_{S}\left(\operatorname{Soc}_{H} A\right)$ is a normal subgroup of $G$ for any normal subgroup $S$ of $G$;

(iii) the quotient group $G / C_{G}\left(\operatorname{Soc}_{H} A\right)$ is abelian-by-finite if the group A has finite rank and the group $G$ is soluble-by-finite.

Proof. It easy to note that the assertions hold for $A$ if and only if they hold for $\bar{A}=A \otimes_{\mathbb{Z}} \mathbb{Q}$. So, we can assume that $\bar{A}=A$ and we consider $A$ as a $\mathbb{Q} G$-module.

(i) We show that $S_{0 c_{H}} A$ is a $\mathbb{Q} G$-submodule of $A$. $\operatorname{Soc}_{H} A=\oplus_{i \in I} A_{i}$, where $A_{i}$ are simple $\mathbb{Q} H$-modules. As $H$ is a normal subgroup of $G, B_{i} g \mathbb{Q} H=$ $B_{i} \mathbb{Q}\left(g H g^{-1}\right) g=B_{i} \mathbb{Q} H g=B_{i} g$ for any $\mathbb{Q} H$-submodule $B_{i}$ of $A_{i}$, any $g \in G$ and any $i \in I$. It implies that $A_{i} g$ is a simple $\mathbb{Q} H$-module and hence $A_{i} g \leqslant S o c_{H} A$ for any $g \in G$ and any $i \in I$. Therefore, $\left(\operatorname{Soc}_{H} A\right) g \leqslant \operatorname{Soc}_{H} A$ for any $g \in G$ and hence $S c_{H} A$ is a $G$-invariant subgroup of $G$.

It follows from the definition of $\Delta_{G}(A)$ that the quotient group $H / C_{H}(a \mathbb{Q} H)$ is finite for any $a \in \Delta_{G}(A)$. Then, by Maschke's Theorem, $a \mathbb{Q} H$ is a semisimple $\mathbb{Q} H$-module for any $a \in \Delta_{G}(A)$. Therefore, $\Delta_{G}(A) \leqslant S o c_{H} A$. Since $\operatorname{Soc}_{H} A$ is a semi-simple $\mathbb{Q} H$-module, we have $\operatorname{Soc}_{H} A=\Delta_{G}(A) \oplus V$, where $V$ is a $\mathbb{Q} H$-module such that $V \cong \operatorname{Soc}_{H} A / \Delta_{G}(A)$. As $|G / H|<\infty$, it is easy to note that $\Delta_{G}(A)=\Delta_{H}(A)$, and hence $\Delta_{H}(V)=0$. Therefore, $\Delta_{H}\left(\operatorname{Soc}_{H} A / \Delta_{G}(A)\right)=\Delta_{G}\left(\operatorname{Soc}_{H} A / \Delta_{G}(A)\right)=0$.

(ii) By (i), $\operatorname{Soc}_{H} A$ is a $G$-invariant subgroup of $G$ and hence for any $h \in C_{S}\left(\operatorname{Soc}_{H} A\right)$, any $g \in G$ and any $a \in S_{S o c_{H}} A$ we have $a h^{g}=\left(a g^{-1}\right) h g=$ $\left(a g^{-1}\right) g=a$ because $a g^{-1} \in S_{S o c} A$. Thus, for any $h \in C_{S}\left(S_{S o c} A\right)$ and any $g \in G$ we have $h^{g} \in C_{S}\left(\operatorname{Soc}_{H} A\right)$ and the assertion follows.

(iii) It follows from the definition of $G$ that $H$ has a soluble $G$-invariant subgroup $S$ of finite index in $G$. As the group $A$ has finite rank, $\operatorname{dim}_{\mathbb{Q}}\left(\operatorname{Soc}_{H} A\right)<$ $\infty$ and hence by Clifford Theorem [24, Theorem 1.7] $S / C_{S}\left(S o c_{H} A\right)$ is a completely reducible soluble subgroup of $G L(n, \mathbb{Q})$. By [24, Theorem 3.5], the 
quotient group $S / C_{S}\left(S_{o c_{H}} A\right)$ is abelian-by-finite. By (ii), $C_{S}\left(S o c_{H} A\right)$ is a $G$-invariant subgroup and, as $|G / S|<\infty$, we see that the quotient group $G / C_{S}\left(\operatorname{Soc}_{H} A\right)$ is abelian-by-finite. As $C_{S}\left(\operatorname{Soc}_{H} A\right) \leqslant C_{G}\left(\operatorname{Soc}_{H} A\right)$, the assertion follows.

Let $A$ be an abelian torsion-free group of finite rank acted by a group $G$. The group $G$ acts on $A$ rationally irreducible if $\bar{A}=A \otimes_{\mathbb{Z}} \mathbb{Q}$ is an irreducible $\mathbb{Q} G$-module and $A$ is said to be a $G$-plinth if any subgroup of finite index in $G$ acts on $A$ rationally irreducible. If $A$ has a finitely generated dense subgroup $B$ such that $B=\oplus_{i=1}^{n} B_{i}$, where $i s_{A}\left(B_{i}\right)$ is a $G$-plinth for each $1 \leqslant i \leqslant n$, then we say that $A$ is a $G$-polyplinth. A $G$-polyplinth is said to be Noetherian if it satisfies the maximal condition for $G$-invariant subgroups.

Lemma 2.4. Let $A$ be an abelian torsion-free group of finite rank acted by a soluble-by-finite group $G$. Then:

(i) $\operatorname{Soc}_{H} A=\operatorname{Soc}_{G} A$ for any normal subgroup $H$ of finite index in $G$;

(ii) $G$ has a normal subgroup $H$ of finite index such that $\operatorname{Soc}_{H} A=\operatorname{Soc}_{G} A$ contains a dense $G$-invariant Noetherian $H$-polyplinth;

(iii) if $\operatorname{Soc}_{G} A=A$ and $A$ satisfies the maximal condition for $G$-invariant subgroups then $\Delta_{G}(A)$ is a finitely generated isolated $G$-invariant subgroup of $A$;

(iv) if $\operatorname{Soc}_{G} A=A$ and $A$ has an isolated $G$-invariant subgroup $B$ such that the quotient group $A / B$ is polycyclic then $A$ has a $G$-invariant polycyclic subgroup $P$ such that $P \cap B=0$ and $P+B$ has finite index in $A$.

Proof. It easy to note that the assertions (i) and (ii) hold for $A$ if and only if they hold for $\bar{A}=A \otimes_{\mathbb{Z}} \mathbb{Q}$. So, in the proof of assertions (i) and (ii) we can assume that $\bar{A}=A$ and we consider $A$ as a $\mathbb{Q} G$-module.

(i) As $\operatorname{dim}_{\mathbb{Q}} A$ is finite, it follows from Clifford Theorem [24, Theorem 1.7] that $\operatorname{Soc}_{X} A \geqslant \operatorname{Soc}_{G} A$ for any normal subgroup $X$ of $G$. Since $\operatorname{dim}_{\mathbb{Q}} A$ is finite, it implies that there is a $G$-invariant subgroup $H$ of finite index in $G$ such that $S_{o c} A=S o c_{S} A$ for any $G$-invariant subgroup $S$ of finite index in $H$. Then it is sufficient to show that $\operatorname{Soc}_{H} A=\operatorname{Soc}_{G} A$. By Lemma 2.3(i,iii), $\operatorname{Soc}_{H} A$ is a $G$-invariant subgroup of $A$ and, the quotient group $G / C_{G}\left(S_{O} c_{H} A\right)$ is abelian-by-finite. Therefore, changing $G$ by $G / C_{G}\left(S o c_{H} A\right)$ we may assume 
that $G$ is abelian-by-finite. So, $G$ has a normal abelian subgroup $X$ of finite index and changing $H$ by $H \cap X$ we may assume that $H$ is abelian.

It is sufficient to show that $\operatorname{Soc}_{H} A$ is a semi-simple $\mathbb{Q} G$-module. Since $H$ is abelian, we see that $\operatorname{Soc}_{H} A=\oplus_{i=1}^{k} B_{i}$, where each $B_{i}$ is a simple $\mathbb{Q} H$ module which is annihilated by a maximal ideal $P_{i}$ of $\mathbb{Q} H$. Since $S_{\text {oc }} A=$ $\sum_{i=1}^{k} B_{i} \mathbb{Q} G$, it is sufficient to show that each $B_{i} \mathbb{Q} G$ is a semi-simple $\mathbb{Q} G$ module. To simplify the denotations, we fix some index $i$ putting $B_{i}=B$ and $P_{i}=P$. Let $K=N_{G}(P)$, evidently $H \leqslant K \leqslant G$ and hence $K$ has finite index in $G$. Since $P$ annihilates $B \mathbb{Q} K$, we can consider $B \mathbb{Q} K$ as a $\mathbb{Q} K / P \mathbb{Q} K$-module. It is easy to note that the quotient ring $\mathbb{Q} K / P \mathbb{Q} K$ is a cross product $F * T$ of the field $F=\mathbb{Q} H / P$ and the group $T=K / H$. So, $B \mathbb{Q} K$ is an $F * T$-module. It follows from [9, Theorem 4.1] that the cross product $F * T$ is a semi-simple ring and hence so is $\mathbb{Q} K / P \mathbb{Q} K$. As $P$ annihilates $B \mathbb{Q} K$, it implies that $B \mathbb{Q} K$ is a semi-simple $\mathbb{Q} K$-module. Thus, $B \mathbb{Q} K=\bigoplus_{i=1}^{r} C_{i}$, where each $C_{i}$ is a simple $\mathbb{Q} K$-module which is annihilated by $P$.

We have to show that $B \mathbb{Q} G$ is a semi-simple $\mathbb{Q} G$-module. Since $B \mathbb{Q} K=$ $\oplus_{i=1}^{r} C_{i}$, we have $B \mathbb{Q} G=\sum_{i=1}^{r} C_{i} \mathbb{Q} G$ and hence it is sufficient to show that $C_{i} \mathbb{Q} G$ is a simple $\mathbb{Q} G$-module for each $i$. To simplify the denotations we fix some index $i$ and put $C=C_{i}$.

Let $T=\left\{t_{1}, \ldots t_{l}\right\}$ be a transversal to $K$ in $G$ then $C \mathbb{Q} G=\sum_{i=0}^{l}(C \mathbb{Q} K) t_{i}$. As $K=N_{G}(P)$, each $\mathbb{Q} H$-submodule $(C \mathbb{Q} K) t_{i}$ is annihilated by $P_{i}=P^{t_{i}}$, where $P_{i}=P^{t_{i}}$ are maximal ideals of $\mathbb{Q} H$ such that $P_{i} \neq P_{j}$ if $i \neq j$.

So, we have to show that $C \mathbb{Q} G$ is a simple $\mathbb{Q} G$-module. As $C$ is a simple $\mathbb{Q} K$-module, it is sufficient to show that $C \cap W \neq 0$ for any $\mathbb{Q} G$-submodule $W \neq 0$ of $C \mathbb{Q} G$. Let $0 \neq a \in W$ then $0 \neq a=\sum_{i=1}^{l} a_{i}$, where $a_{i} \in(C \mathbb{Q} K) t_{i}$ and $a_{i}$ is annihilated by $P_{i}$. If $a_{i} \neq 0$ and $a_{j} \neq 0$, where $i \neq j$, then, as $P_{i} \neq P_{j}$, there is an element $x \in P_{j} \backslash P_{i}$ and hence $a_{i} x \neq 0$ but $a_{j} x=0$. Then the sum $a x=\sum_{i=1}^{l} a_{i} x$, where $a_{i} x \in(C \mathbb{Q} K) t_{i}$, has less non-zero summands than $0 \neq a=\sum_{i=1}^{l} a_{i}$. So, in such way we can decries the number of non-zero summands from $(C \mathbb{Q} K) t_{i}$ in $0 \neq a=\sum_{i=1}^{l} a_{i}$ until we obtain $0 \neq b \in(C \mathbb{Q} K) t_{i} \cap W$ for some $i$. Then $0 \neq b t_{i}{ }^{-1} \in(C \mathbb{Q} K) \cap W$.

(ii) By (i), $\operatorname{Soc}_{H} A=\operatorname{Soc}_{G} A=S$ for any normal subgroup $H$ of finite index in $G$, we denote by $n(H)$ the number of irreducible direct summands in $S_{o c} A=S$. Since the numbers $n(H)$ are bounded by $r(A)$, there is a normal subgroup $H$ of finite index in $G$ with maximal possible $n(H)$. Let $\operatorname{Soc}_{H} A=S=\oplus_{i=1}^{n} S_{i}$, where $S_{i}$ are irreducible $\mathbb{Q} H$-modules, $1 \leqslant i \leqslant n$ and 
$n=n(H)$. Suppose that there is a submodule $S_{i}$ which is not $\mathbb{Q} L$-irreducible for some subgroup $L$ of finite index in $H$. It is well known that there is a normal subgroup $T$ of finite index in $G$ such that $T \leqslant L$ and hence $S_{i}$ is not $\mathbb{Q} T$-irreducible. It easily implies that $S_{i}$ has a proper $\mathbb{Q} T$-irreducible submodule and hence $n(T)>n(H)$ but it contradicts to the choice of the subgroup $H$. Thus, each $S_{i}$ is an irreducible $\mathbb{Q} L$-module for any subgroup $L$ of finite index in $H$ and hence $S_{H} A=S$ is an $H$-polyplinth.

Let $\operatorname{Soc}_{H} A=\operatorname{Soc}_{G} A=S=\oplus_{i=1}^{k} A_{i}$, where $A_{i}$ is an irreducible $\mathbb{Q} G$ module, $1 \leqslant i \leqslant k$. Let $0 \neq b_{i} \in A_{i}, B_{i}=b_{i} \mathbb{Z} G$ and $B=\oplus_{i=1}^{k} B_{i}$. As $A_{i}$ is an irreducible $\mathbb{Q} G$-module, $A_{i}=b_{i} \mathbb{Q} G$ and hence $B=\oplus_{i=1}^{k} B_{i}$ is a dense subgroup of $S_{o c_{G}} A=S=\oplus_{i=1}^{k} A_{i}$. Besides, as $A_{i}$ is an irreducible $\mathbb{Q} G$-module, we see that for any $0 \neq x \in B_{i}$ there is $m \in \mathbb{N}$ such that $b_{i} m \in x \mathbb{Z} G$ and hence $B_{i} m \leqslant x \mathbb{Z} G$. Since $\left|B_{i} / B_{i} m\right|<\infty$, we can conclude that $\left|B_{i} / x \mathbb{Z} G\right|<\infty$ for any $0 \neq x \in B_{i}$ and it easily implies that $B_{i}$ is a Noetherian $\mathbb{Z} G$-module for each $1 \leqslant i \leqslant n$ and hence so is $B=\oplus_{i=1}^{k} B_{i}$. It follows from [22, Theorem A] that $B=\oplus_{i=1}^{k} B_{i}$ meets the maximal condition for $H$-invariant subgroups. As $\operatorname{Soc}_{H} A=S$ is an $H$-polyplinth and $B=$ $\oplus_{i=1}^{k} B_{i}$ is dense in $\operatorname{Soc}_{H} A=S$, we can conclude that $B=\oplus_{i=1}^{k} B_{i}$ is a $G$-invariant dense in $S$ Noetherian $H$-polyplinth.

(iii) Since $r(A)<\infty$, it follows from the definition of $\Delta_{G}(A)$ that it has a finitely generated dense $G$-invariant subgroup $D$. As $A$ satisfies the maximal condition for $G$-invariant subgroups, it implies that $\left|\Delta_{G}(A) / D\right|<\infty$ and hence $\Delta_{G}(A)$ is a finitely generated subgroup.

(iv) Let $\bar{A}=A \otimes_{\mathbb{Z}} \mathbb{Q}$ and $\bar{B}=B \otimes_{\mathbb{Z}} \mathbb{Q}$. Since $\operatorname{Soc}_{G} A=A$, we see that $\bar{A}$ is a semi-simple $\mathbb{Q} G$-module and hence there is a submodule $\bar{P}$ of $\bar{A}$ such that $\bar{P} \cap \bar{B}=0$ and $\bar{P}+\bar{B}=\bar{A}$. Put $P=\bar{P} \cap A$ then $P \cap B=0$ and $P+B$ is dense in $A$. As the quotient group $A / B$ is polycyclic, we can conclude that $P$ is a $G$-invariant polycyclic subgroup of $A$ and $P+B$ has finite index in $A$.

Let $R$ be a ring, an $R$-module is said to be uniform if any its two non-zero submodules have non-zero intersection.

Lemma 2.5. Let $G$ be a group, let $N$ be a normal subgroup of $G$. Let $R$ be a domain and let $0 \neq W$ be an $R G$-module. Suppose that the subgroup $N$ is torsion-free nilpotent. If $A n n_{S N} w \neq 0$ for some $0 \neq w \in W$ and some subring $S \leqslant R$ then the submodule $w R G$ is $S N$-torsion.

Proof. Any element $0 \neq x \in w R G$ may be presented in the form $x=$ 
$\sum_{i=1}^{m} w d_{i} g_{i}$, where $d_{i} \in R, g_{i} \in G$ and $m \in \mathbb{N}$. Put $J=A n n_{S X} w \neq 0$, it follows from [9, Corollary 37.11] that the ring $S N$ is an Ore domain and hence $S N$ is uniform. Therefore, $I=\cap_{i=1}^{m} g_{i}^{-1} J g_{i} \neq 0$. Evidently, $I \leqslant A n n_{S N}(x)$ and hence the submodule $w R G$ is $S N$-torsion.

\section{Large Culling Ideals of Group Rings of Min- imax Nilpotent Groups}

Let $K$ be a normal subgroup of a group $L$ such that the quotient group $L / K$ is free abelian with free generators $K x_{1}, K x_{2}, \ldots, K x_{n}$. We say that $\chi=\left\{\left\langle K,\left\{x_{j} \mid j \in J\right\}\right\rangle \mid J \subseteq\{1, \ldots, n\}\right\}$ is a full system of subgroups of $L$ over $K$.

Let $k$ be a field, let $V$ be a $k K$-module and let $W$ be an image of $V \otimes_{k K} k L$ under a $k L$-module homomorphism $\alpha$. Put

$$
\chi(W)=\left\{X \in \chi \mid \operatorname{ker} \alpha \cap\left(V \otimes_{k K} k X\right\}=0\right\}
$$

and $M \chi(W)$ denotes the set of maximal elements of $\chi(W)$.

Lemma 3.1. Let $L$ be a minimax nilpotent group and $K$ be a normal subgroup of $L$ such that the quotient group $L / K$ is free abelian with free generators $K x_{1}, K x_{2}, \ldots, K x_{n}$. Let $\chi=\left\{\left\langle K,\left\{x_{j} \mid j \in J\right\}\right\rangle \mid J \subseteq\{1, \ldots, n\}\right\}$ be a full system of subgroups of $L$ over $K$. Then the group $L$ has a finitely generated dense subgroup $H$ such that if a subgroup $H_{1} \leqslant L$ contains $H$ then for any $L$-invariant subgroup $I^{\dagger} \leqslant K$ of finite index in $K$

(i) $K=I^{\dagger} K_{1}$ and $L=I^{\dagger} H_{1}$, where $K_{1}=K \cap H_{1}$;

(ii) $\chi=\left\{I^{\dagger} X \mid X \in \chi_{H}\right\}$, where $\chi_{H}=\left\{\left\langle K_{1},\left\{x_{j} \mid j \in J\right\}\right\rangle \mid J \subseteq\{1, \ldots, n\}\right\}$ is a full system of subgroups of $H_{1}$ over $K_{1}=K \cap H_{1}$.

Proof. Since the group $L$ is nilpotent and minimax, the subgroup $K$ has a finite central series

$$
\langle 1\rangle=C_{0} \leqslant C_{1} \leqslant \ldots \leqslant C_{n}=K
$$

all of whose quotients are cyclic or quasi-cyclic. Let $\left\{y_{1}, \ldots, y_{m}\right\}$ be a set

of elements of $K$ which generate all cyclic quotients of (5) and let $H$ be a 
subgroup of $L$ generated by $\left\{x_{1}, x_{2}, \ldots, x_{n}\right\} \cup\left\{y_{1}, \ldots, y_{m}\right\}$. Then $H$ is a finitely generated dense subgroup of $L$. Let $H_{1}$ be a subgroup of $L$ such that $H \leqslant H_{1}$.

(i) Since $\left\{y_{1}, \ldots, y_{m}\right\} \subseteq K_{1}=K \cap H_{1}$, all non-trivial quotients of the series

$$
K_{1}=C_{0} K_{1} \leqslant C_{1} K_{1} \leqslant \ldots \leqslant C_{n}=K
$$

are quasi-cyclic and hence divisible. Let $I^{\dagger}$ be an $L$-invariant subgroup of finite index in $K$, as $K_{1}$ meets the conditions of [18, Lemma 2.1.7], we have $K=I^{\dagger} K_{1}$. As $\left\{x_{1}, x_{2}, \ldots, x_{n}\right\} \subseteq H_{1}$, we have $L=K H_{1}$ and hence $L=$ $K H_{1}=\left(I^{\dagger} K_{1}\right) H_{1}=I^{\dagger}\left(K_{1} H_{1}\right)=I^{\dagger} H_{1}$.

(ii) It follows from the definitions of $\chi$ and $\chi_{H}$ that $\chi=\left\{K X \mid X \in \chi_{H}\right\}$. Then, as $K=I^{\dagger} K_{1}$ and $K_{1} \leqslant X$ for any $X \in \chi_{H}$, we have $\chi=\left\{K X \mid X \in \chi_{H}\right\}=$ $\left\{\left(I^{\dagger} K_{1}\right) X \mid X \in \chi_{H}\right\}=\left\{I^{\dagger}\left(K_{1} X\right) \mid X \in \chi_{H}\right\}=\left\{I^{\dagger} X \mid X \in \chi_{H}\right\}$.

Lemma 3.2. Let $N$ be a minimax nilpotent torsion-free group and let $K$ be a normal subgroup of $N$ such that the quotient group $N / K$ is torsion-free abelian. Let $L$ be a normal subgroup of $N$ such that $K \leqslant L$ and the quotient group $L / K$ is free abelian. Let $\chi$ be a full system of subgroups of $L$ over $K$. Let $k$ be a field and let $0 \neq W$ be a $k N$-module which is $k K$-torsionfree. Then there is a $k N$-submodule $0 \neq V \leqslant W$ such that for any element $0 \neq a \in V:$

(i) there is a finitely generated dense subgroup $H \leqslant L$ such that akL $=$ $a k H \otimes_{k H} k L$;

(ii) the module $V$ is $k X$-torsion-free for any $X \in \chi(a k L)$ and $k X$-torsion for any $X \in \chi \backslash \chi(a k L)$;

(iii) $\chi(a k L)=\chi(b k L)$ for any element $0 \neq b \in V$;

(iv) in the case where $N=L$, for any subgroup $X \in M \chi(a k L)$ we have $b k L \cap a k(X \cap H) \neq 0$ for any element $0 \neq b \in a k L$ and any finitely generated dense subgroup $H \leqslant L$. In particular, bkL $\cap$ akH $\neq 0$, $b k L \cap a k X \neq 0$ and the module akL is uniform.

Proof. It is easy to note that in the proof the module $W$ may be changed by any its proper submodule.

(i). By [18, Lemma 2.2.5], the assertion holds for any element $0 \neq a \in W$.

(ii). Let $X \in \chi$ if the module $W$ is $k X$-torsion-free then $X \in \chi(a k L)$ for any element $0 \neq a \in W$. If $A n n_{k X} w \neq 0$ for some $0 \neq w \in W$ then we may 
change $W$ by $w k N$. As $X$ is a normal subgroup of $N$, it follows from Lemma 2.5 that the module $V=w k N$ is $k X$-torsion and hence $X \in \chi \backslash \chi(a k L)$ for any element $0 \neq a \in V$. Taking $Y \in \chi \backslash\{X\}$ and repeating the above arguments we obtain a $k N$-submodule $0 \neq V_{1} \leqslant V$ which is either $k Y$-torsion or $k Y$-torsion-free. Continuing this process we see that it is terminated because the set $\chi$ is finite.

(iii). The assertion follows from (ii).

(iv). By (ii), we can assume that for any $0 \neq w \in W$ and $X \in M \chi(w R L)$ the $k N$-module $w k N$ is $k X$-torsion-free and hence, by [18, Lemma 2.2.4(ii)], there exists a $k L(k X)^{-1}$-module $w k L(k X)^{-1}$. Then it easily follows from the maximality of $X$ that $w k L(k X)^{-1}$ has finite dimension over the field $k X(k X)^{-1}$. Therefore, we can choose the element $0 \neq v \in w k L$ such that $v k L(k X)^{-1}$ is a simple $k L(k X)^{-1}$-module. Put $V_{1}=v k L$. Then for any $0 \neq$ $a, b \in V$ we have $V=a k L(R X)^{-1}=b k L(R X)^{-1}$ and hence $a \in b k L(k X)^{-1}$, it easily implies that $b k L \cap a k X \neq 0$. Since $H \cap X$ is a dense subgroup of $X$, it follows from [18, Lemma 2.2.3(ii)] that $b k L \cap a k(H \cap X) \neq 0$. The last equations also shows that $b k L \cap a k H \neq 0$ and the module $V_{1}=v k L$ (and hence any its proper submodule) is uniform. Thus, we obtained a submodule $0 \neq V_{1}=v k L$ such that the assertion (iv) holds for the chosen subgroup $X \in M \chi(v R L)$ and elements of $V_{1}$.

Suppose that there is a subgroup $Y \in M \chi(v R L) \backslash\{X\}$. Applying the above arguments to $Y$ and the submodule $V_{1}$ we may obtain a submodule $0 \neq V_{2} \leqslant V_{1}$ such that the assertion (iv) holds for the chosen subgroups $X, Y \in M \chi(v R L)$ and elements of $V_{2}$. Continuing this process we see that it is terminated because the set $\chi$ is finite.

Let $G$ be a group and let $K$ be a normal subgroup of $G$. Let $R$ be a ring and let $I$ be a $G$-invariant ideal of the group $\operatorname{ring} R K$ then $I^{\dagger}=$ $G \bigcap(I+1)$ is a $G$-invariant subgroup of $G$. We say that the ideal $I$ is $G$ large if $R /(R \bigcap I)=k$ is a field, $\left|K / I^{\dagger}\right|<\infty$ and $I=(R F \bigcap I) R K$, where $F$ is a $G$-invariant subgroup of $K$ such that $I^{\dagger} \leqslant F$ and the quotient group $F / I^{\dagger}$ is abelian. If $R$ is a field then, certainly, $R=k$. If $N$ is a subgroup of $G$ such that $K \leqslant N$ and $F / I^{\dagger}$ is a central section of $N$ then we say that the ideal $I$ is $N$-central.

Lemma 3.3. Let $K$ be a normal subgroup of a torsion-free minimax nilpotent group $N$ such that the quotient group $N / K$ is torsion-free abelian. Let $L$ be a dense subgroup of $N$ such that $K \leqslant L$ and the quotient group $L / K$ is free abelian. Let $k$ be a field, let $I$ be an $N$-large ideal of $k K$ and let $A \leqslant L / I^{\dagger}$ 
be a central dense free subgroup of $N / I^{\dagger}$. Let $0 \neq W$ be a $k N$-module which is $k K$-torsion-free. Then there is a $k L$-submodule $0 \neq V \leqslant W$ such that for any element $0 \neq a \in V$ and any element $0 \neq b \in a k N$ :

(i) bkL is not isomorphic to any proper section of agkL for any $g \in N$;

(ii) the $k A$-module bkL/bkLI has a finite series each of whose quotient is isomorphic to some section of the $k A$-module akL/akLI;

(iii) there is a kL-submodule $0 \neq c k L \leqslant$ akL such that the $k A$-module ckL/ckLI has a finite series each of whose quotient is isomorphic to some section of the $k A$-module bkL/bkLI.

Proof. (i) By Lemma 3.2(ii), we can assume that for any element $0 \neq a \in W$ and any subgroup $X \in M \chi(a k L)$ the module $W$ is $k X$-torsion-free. By Lemma 3.2(iv), there is a $k L$-submodule $0 \neq V \leqslant W$ such that for any element $0 \neq a \in V$ and any element $0 \neq b \in a k L$ we have $b k L \cap a k X \neq 0$. Therefore, for any submodule $0 \neq U \leqslant a k L$ the quotient module $a k L / U$ is $k X$-torsion. As $K \leqslant X$ and the quotient group $N / K$ is abelian, $X$ is a normal subgroup of $N$ and hence $a g k L / U$ is $k X$-torsion for any $k L$-submodule $0 \neq$ $U \leqslant a g k L$ and any element $g \in N$. It easily implies that any proper section of $a g k L$ is $k X$-torsion. On the other hand, the module $a k N$ is $k X$-torsion-free and the assertion follows.

(ii). Step 1. At first, we prove the assertion in the case, where $0 \neq b \in$ $a k L$. By [18, Lemma 2.2.5], there is a finitely generated dense subgroup $H \leqslant L$ such that $a k L=a k H \otimes_{k H} k L$. Evidently, the subgroup $H$ can be chosen such that $0 \neq b \in a k H$ and $I^{\dagger} H=L$ then $b k L=b k H \otimes_{k H} k L$.

Put $K_{1}=K \cap H$ and $I_{1}=I \cap k K_{1}$ then it follows from [18, Lemma 2.2.6] that $a k L / a k L I \cong a k H / a k H I_{1}$ and $b k L / b k L I \cong b k H / b k H I_{1}$ considered as $k A$-modules, where $A$ is a central subgroup of finite index in $L / I^{\dagger}$. Therefore, it is sufficient to show that the $k A$-module $b k H / b k H I_{1}$ has a finite series each of whose quotient is isomorphic to some section of the $k A$-module $a k H / a k H I_{1}$.

Since the ideal $I_{1}$ is $H$-large, there exists an $H$-invariant subgroup $F \leqslant$ $K_{1}$ such that the quotient group $F / I_{1}^{\dagger}$ is abelian and $I_{1}=\left(k F \bigcap I_{1}\right) k K_{1}$. As $\left|F / I_{1}^{\dagger}\right|<\infty$, we have $|H: C|<\infty$, where $C=C_{H}\left(F / I_{1}^{\dagger}\right)$. The arguments of the proof of [18, Lemma 2.4.1(i)] shows that $\left(k F \bigcap I_{1}\right) k C$ is a polycentral 
ideal of $k C$. As $|H: C|<\infty$, we see that akH is a finitely generated $k C$ module and, as $I_{1}=\left(k F \bigcap I_{1}\right) k K_{1}$, we can conclude that $a k H\left(k F \bigcap I_{1}\right) k C=$ $a k H I_{1}$. Then the assertion follows from [18, Lemma 2.4.1(ii)].

Step 2. Consider now the general case. The element $b$ may be written in the form $b=a_{1} g_{1}+\ldots+a_{n} g_{n}$, where $a_{i} \in a k L$ and $g_{i} \in N$. Then it is easy to note that $b k L \cong\left(\oplus_{i=1}^{n} a_{i} k L g_{i}\right) / U$, where $U$ is a $k L$-submodule of $\oplus_{i=1}^{n} a_{i} g_{i} k L$, and hence $b k L / b k L I \cong\left(\oplus_{i=1}^{n}\left(a_{i} k L / a_{i} k L I\right) g_{i}\right) / U_{1}$, where $U_{1}$ is a $k L$-submodule of $\oplus_{i=1}^{n}\left(a_{i} k L / a_{i} k L I\right) g_{i}$. Since $A$ is a central subgroup of $N / I^{\dagger}$ , we see that $\left.\left(a_{i} k L / a_{i} k L I\right) g_{i}\right) \cong a_{i} k L / a_{i} k L I$ considered as $k A$-modules and hence $b k L / b k L I \cong\left(\oplus_{i=1}^{n}\left(a_{i} k L / a_{i} k L I\right)\right) / U_{1}$, where $U_{1}$ is a $k L$-submodule of $\oplus_{i=1}^{n}\left(a_{i} k L / a_{i} k L I\right)$. As it proved in step $1, a_{i} k L / a_{i} k L I$ has a finite series each of whose quotient is isomorphic to some section of $a k L / a k L I$ and the assertion follows.

(iii). Step 1. At first, we show that $b k L$ contains a submodule $d k L$ isomorphic to $c g k L=c k L g$, where $0 \neq c \in a k L$ and $g \in N$. The element $b$ may be written in the form $b=a_{1} g_{1}+\ldots+a_{n} g_{n}$, where $a_{i} \in a k L$ and $g_{i} \in N$. The proof is by induction on $n$.

Evidently, $b k L+\left(a_{2} g_{2}+\ldots a_{n} g_{n}\right) k L=a_{1} g_{1} k L+\left(a_{2} g_{2}+\ldots a_{n} g_{n}\right) k L$ and hence $b k L+\left(a_{2} g_{2}+\ldots a_{n} g_{n}\right) k L /\left(a_{2} g_{2}+\ldots a_{n} g_{n}\right) k L=a_{1} g_{1} k L+\left(a_{2} g_{2}+\ldots a_{n} g_{n}\right) k L /\left(a_{2} g_{2}+\right.$ $\left.\ldots a_{n} g_{n}\right) k L$. If $b k L \cap\left(a_{2} g_{2}+\ldots a_{n} g_{n}\right) k L=0$ then $b k L \cong a_{1} g_{1} k L /\left(a_{1} g_{1} k L \cap\right.$ $\left.\left(a_{2} g_{2}+\ldots a_{n} g_{n}\right) k L\right)$. Then it follows from (i) that $a_{1} g_{1} k L \cap\left(a_{2} g_{2}+\ldots a_{n} g_{n}\right) k L=$ 0 and hence $b k L \cong a_{1} g_{1} k L$. So, we can put $c=a_{1}$ and $g=g_{1}$.

If there is $0 \neq b_{1} \in b k L \cap\left(a_{2} g_{2}+\ldots a_{n} g_{n}\right) k L$ then $b_{1}$ can be written in the form $b_{1}=e_{2} g_{2}+\ldots e_{n} g_{n} k L$ where $e_{i} \in a k L$. As $0 \neq b_{1} k L \leqslant b k L$, changing the element $b$ by $b_{1}$ we can apply the induction hypothesis.

Step 2. By (ii), $d k L / d k L I$ has a finite series each of whose quotient is isomorphic to some section of $b k L / b k L I$ considered as $k A$-modules and it follows from step 1 that $c k L g / c k L g I$ has such a series. Since $A$ is a central subgroup of $N / I^{\dagger}$, we can conclude that $c k L g / c k L g I$ and $c k L / c k L I$ are isomorphic as $k A$-modules.

Let $R$ be a ring, let $V$ be an $R$-module and $U$ be a submodule of $V$. An ideal $I$ of $R$ culls $U$ in $V$ if $V I<V$ and $V I+U=V$ (see [4]).

Lemma 3.4. Let $R$ be a ring, let $V$ be an $R$-module and $U$ be a submodule of $V$. An ideal $I$ of $R$ culls $U$ in $V$ if and only if $I^{n}$ culls $U$ in $V$ for any $n \in \mathbb{N}$.

Proof. If $V I<V$ then $V I^{n}<V$ for any $n \in \mathbb{N}$. If $V I+U=V$ then 
$V I^{2}+U I=V I$ and hence $V I^{2}+U=V I^{2}+U I+U=V I+U=V$. Thus, $V I^{2}+U=V$ and by induction we can conclude that $V I^{n}+U=V$ for any $n \in \mathbb{N}$.

Let $R$ be a domain, $K$ be a normal subgroup of a group $H$. According to [4, Introduction, p. 89], we say that that an $R K$-module $0 \neq V$ is $H$-ideal critical if for any submodule $0 \neq V_{1} \leqslant V$ there is an $H$-invariant ideal $I$ of $R K$ such that:

(i) $I$ culls $V_{1}$ in $V$;

(ii) $I$ has the weak Artin-Rees property that is, if $U$ is any finitely generated $R K$-module with submodule $U_{1}$ then $U I^{n} \cap U_{1} \leqslant U_{1} I$ for some $n \in \mathbb{N}$ dependent on $U$ and $U_{1}$.

Proposition 3.1. Let $N$ be a minimax nilpotent torsion-free normal subgroup of a soluble group $G$ such that $r_{0}(G)<\infty$ and let $K$ be a $G$-invariant subgroup of $N$. Let $H$ be a finitely generated subgroup of $K$, let $k$ be a field of characteristic zero and let $0 \neq J$ be a right ideal of $k H$. Then:

(i) there is a $G$-large $N$-central ideal $I$ of $k K$ such that $k H \cap I$ culls $J$ in $k H$;

(ii) if the group $G=N$ then the ideal $I$ may be chosen such that $I=$ $(I \cap k F) k K$, where $K \geqslant F \geqslant I^{\dagger}, F / I^{\dagger}$ is a central section of $G$ and $I \cap k F$ is a maximal ideal of $k F$

(iii) if $H=N$ then $k K$ is $H$-ideal critical uniform $k K$-module.

Proof. (i), (ii). By [25, Theorem 3.7], the ring $k H$ is Noetherian and hence the ideal $J$ is finitely generated. Therefore, there is a finitely generated subfield $k_{1} \leqslant k$ such that $J=\left(J \cap k_{1} H\right) k H$. By [18, Proposition 2.4.3], there is a $G$-large $N$-central ideal $I_{1}$ of $k_{1} K$ such that $k_{1} H \cap I_{1}$ culls $J \cap k_{1} H$ in $k_{1} H$ and if $G=N$ then the ideal $I_{1}$ may be chosen such that $I_{1}=\left(I_{1} \cap k_{1} F\right) k_{1} K$, where $K \geqslant F \geqslant I_{1}^{\dagger}, F / I_{1}^{\dagger}$ is a central section of $G$ and $I_{1} \cap k_{1} F$ is a maximal ideal of $k_{1} F$. Put $I=\left(I_{1} \cap k_{1} F\right) k K$ then $k H \cap I$ culls $J$ in $k H$. If $G=N$ and $I \cap k F$ is not a maximal ideal of $k F$ then we can change $I \cap k F$ by any maximal ideal $P$ of $k F$ containing $I \cap k F$ and put $I=P k K$.

(iii). By [18, Lemma 2.4.1(i)], any $H$-large ideal $I$ of $k K$ is polycentral and it follows from [25, Theorem 6.12] that $I$ has the weak Artin-Rees property. Then (i) implies that $k K$ is $H$-ideal critical. It follows from [9, Corollary $37.11]$ that $k K$ is an Ore domain and hence $k K$ is uniform. 
Let $K$ be a normal subgroup of a group $N$ such that the quotient group $N / K$ is torsion-free abelian of finite rank. Let $I^{\dagger}$ be an $N$-invariant subgroup of $K$ such that the quotient group $K / I^{\dagger}$ is finite. As the quotient group $K / I^{\dagger}$ is finite and the quotient group $N / K$ is torsion-free abelian of finite rank, it follows from Lemma 2.2(i) that $N / I^{\dagger}$ has a torsion-free abelian characteristic central subgroup $A$ of finite index. For any subgroup $X$ of $N$ such that $K \leqslant X$ we denote $A_{X}=A \cap X / I^{\dagger}$.

Lemma 3.5. Let $K$ be a normal subgroup of a torsion-free finitely generated nilpotent group $H$ such that the quotient group $H / K$ is free abelian and let $\chi$ a full system of subgroups of $H$ over $K$. Let $k$ be a field and let $0 \neq W$ be a $k H$-module which is $k K$-torsion-free. Then :

(i) for any element $0 \neq a \in W$ there is a right ideal $J_{0}$ of $k K$ such that if $I$ is an $H$-large ideal of $k K$ which culls $J_{0}$ in $k K$ then $X \in \chi(a k H)$ if and only if the quotient module akH/akHI is not $k A_{X}$-torsion, where $A$ is a characteristic central torsion-free subgroup of finite index in $H / I^{\dagger}$ and $A_{X}=A \cap X / I^{\dagger}$;

(ii) for any element $0 \neq a \in W$ any subgroup $X \in \chi(a k H)$ there is a right ideal $J_{X}$ of $k K$ such that if $I$ is an $H$-large ideal of $k K$ which culls $J_{X}$ in $k K$ then for any element $0 \neq c \in a k X$ the quotient module $c k H / c k H I$ is not $k A_{X}$-torsion, where $A$ is a characteristic central torsion-free subgroup of finite index in $H / I^{\dagger}$ and $A_{X}=A \cap X / I^{\dagger}$;

(iii) there are a cyclic $k H$-submodule $0 \neq a k H \leqslant W$ and a right ideal $J$ of $k K$ such that if $I$ is an $H$-large ideal of $k K$ which culls $J$ in $k H$ then for any cyclic $k H$-submodule $0 \neq b k H \leqslant a k H$ we have $X \in \chi(b k H)$ if and only if the quotient module $b k H / b k H I$ is not $k A_{X}$-torsion, where $A$ is a characteristic central torsion-free subgroup of finite index in $H / I^{\dagger}$ and $A_{X}=A \cap X / I^{\dagger}$.

Proof. (i) Since the module $0 \neq W$ is $k K$-torsion-free, $V=a k K \cong k K_{k K}$ is an $H$-ideal critical $k K$-module by Proposition 3.1(iii).

It follows from [4, Lemma 8] that there is a right ideal $J_{0}$ of $k K$ such that if an $H$-large ideal $I$ of $k K$ culls $J_{0}$ in $k K$ (i.e. $I$ culls $a J_{0}$ in $V=a k K \cong k K_{k K}$ ) then $X \in \chi(b k H)$ if and only if $b k H / b k H I \otimes_{k A_{X}} Q_{X} \neq 0$, where $Q_{X}$ denotes the field of fractions of the domain $k A_{X}$. It is well known that the relation $(b k H / b k H I) \otimes_{k A_{X}} Q_{X} \neq 0$ means that the quotient module $b k H / b k H I$ is not $k A_{X}$-torsion. 
(ii) Let $X \in \chi(a k H)$, arguments of the proof of [4, Lemma 14] show that there is a right ideal $J_{X} \leqslant k K$ such that if an $H$-large ideal $I$ of $k K$ culls $J_{X}$ in $k K$ then $a k X I=a k H I \cap a k X$. By Lemma 3.4, $I^{n}$ also culls $J_{X}$ and we can conclude that

$$
a k X I^{n}=a k H I^{n} \cap a k X .
$$

It follows from the definition of $M \chi(a k H)$ that $a k X \cong k X$ and it easily follows from Proposition 3.1(iii) that $\cap_{n \in N} a k X I^{n}=0$. Then there is $m \in \mathbb{N}$ such that $c \in a k X I^{m-1} \backslash a k X I^{m}$. Therefore, $c k X /\left(c k X \cap a k X I^{m}\right) \cong(c k X+$ $\left.a k X I^{m}\right) / a k X I^{m}$ is a non-zero submodule of $a k X I^{m-1} / a k X I^{m}$.

Show now that $S=k X I^{m-1} / k X I^{m}$ is a torsion-free $k A_{X}$-module and hence so is $a k X I^{m-1} / a k X I^{m}$. Evidently, $S=\left(I^{m-1} / I^{m}\right) \otimes_{k C} k B$, where $C=K / I^{\dagger}$ and $B=X / I^{\dagger}$. Then, as $A \cap C=1, S$ is $k A_{X}$-torsion-free.

Thus, $a k X I^{m-1} / a k X I^{m}$ is a $k A_{X}$-torsion-free module and hence so is $\left(c k X+a k X I^{m}\right) / a k X I^{m} \cong c k X /\left(c k X \cap a k X I^{m}\right)$. Due to (6) , as $c \in a k X$, we see that $a k X I^{n} \cap c k X=a k H I^{n} \cap c k X$ and hence $c k X /\left(c k X \cap a k H I^{m}\right)$ is a $k A_{X}$-torsion-free module. As $c k X \leqslant a k X I^{m-1}$, we see that $c k H \leqslant a k H I^{m-1}$ and hence $c k H I \leqslant a k H I^{m}$. It implies that $c k X \cap c k H I \leqslant c k X \cap a k H I^{m}$ and , as $c k X /\left(c k X \cap a k H I^{m}\right)$ is a $k A_{X}$-torsion-free module, the quotient module $c k X /(c k X \cap c k H I) \simeq(c k X+c k H) / c k H I \leqslant c k H / c k H I$ is not $k A_{X}$-torsion.

(iii). We apply Lemma 3.2 to the module $W$ in the case, where $L=H$. Then we can choose an element $0 \neq a \in W$ such that for any $0 \neq b \in a k H$ the following relations hold:

$$
\chi(a k H)=\chi(b k H)
$$

and

$$
a k X \cap b k H \neq 0
$$

for any $X \in M \chi(a k H)$.

Let $J_{0}$ be a right ideal of $k K$ from (i) defined for the element $a$ and let $J_{X}$ be a right ideal of $k K$ from (ii) defined for the element $a$ and for a subgroup $X \in \chi(a k H)$. Put $J=J_{0} \cap\left(\cap_{X \in \chi(a k H)} J_{X}\right)$ and let $I$ be an $H$-large ideal of $k K$ which culls $J$ in $k K$ then, by [4, Lemma 6], $I$ culls $J_{0}$ and $J_{X}$ in $k K$ for each $X \in \chi(a k H)$. Therefore, (i) and (ii) hold for the module $a k H$ and the ideal $I$.

Let $0 \neq b k H \leqslant a k H$ and suppose that for some $X \in \chi$ the quotient module $b k H / b k H I$ is not $k A_{X}$-torsion. Then it follows from Lemma 3.3(ii) that the quotient module $a k H / a k H I$ is not $k A_{X}$-torsion and by (i) $X \in$ $\chi(a k H)$. Therefore, by (7), $X \in \chi(b k H)$. 
Let now $X \in \chi(b k H)$ and show that the quotient module $b k H / b k H I$ is not $k A_{X}$-torsion. Evidently, $X \leqslant Y$ for some $Y \in M \chi(b k H)$ and if we show that $b k H / b k H I$ is not $k A_{Y}$-torsion then $b k H / b k H I$ is not $k A_{X}$-torsion because $A_{X} \leqslant A_{Y}$. Thus, we can assume that $X \in M \chi(b k H)$ and hence, by (8), there is $0 \neq c \in a k X \cap b k H$. As $0 \neq c \in a k X$, it follows from (ii) that the quotient module $c k H / c k H I$ is not $k A_{X}$-torsion. Then, as $0 \neq c k H \leqslant b k H$, it follows from Lemma 3.3(ii) that the quotient module $b k H / b k H I$ is not $k A_{X}$-torsion.

Proposition 3.2. Let $K$ be a normal subgroup of a torsion-free minimax nilpotent group $N$ such that the quotient group $N / K$ is torsion-free abelian. Let $L$ be a dense subgroup of $N$ such that $K \leqslant L$ and the quotient group $L / K$ is free abelian. Let $k$ be a field and let $0 \neq W$ be a $k N$-module which is $k K$-torsion-free. Then there are an element $0 \neq a \in W$, a finitely generated dense subgroup $H$ of $L$ and a right ideal $J$ of $k(H \cap K)$ such that if $I$ is an $N$-large ideal of $k K$ such that $I \cap k(K \cap H)$ culls $J$ in $k(H \cap K)$ then for any $0 \neq b \in a k N$ we have $X \in \chi(b k L)$ if and only if the quotient module $b k L / b k L I$ is not $k A_{X}$-torsion, where $A_{X}=A \cap X / I^{\dagger}$ and $A$ is a characteristic central torsion-free subgroup of finite index in $N / I^{\dagger}$.

Proof. By Lemmas 3.2 and 3.3, we can choose a cyclic submodule $0 \neq a k N \leqslant$ $W$ which satisfies the conditions (i)-(iv) of Lemma 3.2 and the conditions (ii),(iii) of Lemma 3.3 and these conditions hold for any cyclic submodule $0 \neq b k N \leqslant a k N$.

Step 1. Consider at first the case where $N=L$. By Lemma 3.2(i), there is a finitely generated dense subgroup $H$ of $L$ such that

$$
a k L=a k H \otimes_{k H} k L .
$$

It follows from Lemma 3.1 that the subgroup $H$ may be chosen such that for any $L$-invariant subgroup $I^{\dagger} \leqslant K$ of finite index in $K$

$$
I^{\dagger} H=L
$$

and

$$
\chi=\left\{I^{\dagger} X \mid X \in \chi_{H}\right\},
$$

where $\chi_{H}=\left\{\left\langle K_{1},\left\{x_{j} \mid j \in J\right\}\right\rangle \mid J \subseteq\{1, \ldots, n\}\right\}$ is a full system of subgroups of $H$ over $K_{1}=K \cap H$.

Show that

$$
\chi(a k L)=\left\{I^{\dagger} X \mid X \in \chi_{H}(a k H)\right\} .
$$


We use Eq. (11). Let $X \in \chi_{H}(a k H)$ if $I^{\dagger} X \in \chi \backslash \chi(a k L)$ then, by Lemma 3.2 (ii), the module $a k L$ is $k\left(I^{\dagger} X\right)$-torsion and, as $X$ is a dense subgroup of $I^{\dagger} X$, it follows from [18, Lemma 2.2.3(ii)] that the module $a k L$ is $k X$ torsion. But then $X \notin \chi_{H}(a k H)$ and a contradiction is obtained. Suppose now that $I^{\dagger} X \in \chi(a k L)$, where $X \in \chi_{H}$ then, by Lemma 2.3(ii), the module $a k L$ is $k\left(I^{\dagger} X\right)$-torsion-free. Therefore, $a k H$ is $k X$-torsion-free and hence $X \in \chi_{H}(a k H)$. So, the relation (12) holds.

By Lemma 3.5(iii), we can choose the element $a$ for which there is a right ideal $J$ of $k K_{1}$ such that if $I_{1}$ is an $H$-large ideal of $k K$ which culls $J$ in $k K_{1}$ then for any cyclic $k H$-submodule $0 \neq b k H \leqslant a k H$ we have $X \in \chi_{H}(b k H)$ if and only if the quotient module $b k H / b k H I_{1}$ is not $k A_{X}$-torsion, where $A_{X}=A \cap X / I_{1}{ }^{\dagger}$ and $A$ is a central subgroup of finite index in $H / I^{\dagger}$.

Let $I$ be an $L$-large ideal of $k K$ such that $I_{1}=I \cap k K_{1}$ culls $J$ in $k K_{1}$. It follows from (10) and [18, Lemma 2.2.6] that $a k L / a k L I$ and $a k H / a k H I_{1}$ are isomorphic as $k A$-modules, where $A$ is a central subgroup of finite index in $L / I^{\dagger}$. Then it follows from Lemma 3.5(iii) and (12) that $X \in \chi(a k L)$ if and only if the quotient module $a k L / a k L I$ is not $k A_{X}$-torsion, where $A_{X}=A \cap X / I^{\dagger}$ and $A$ is a central subgroup of finite index in $L / I^{\dagger}$.

Let $0 \neq b \in a k L$, by Lemma 3.2(iv), there is an element $0 \neq c \in b k L \cap a k H$ and it follows from (9) that we have a chain of submodules $c k H \otimes_{k H} k L=$ $c k L \leqslant b k L \leqslant a k L=a k H \otimes_{k H} k L$. By Lemma 3.2(iii), $\chi(c k L)=\chi(b k L)=$ $\chi(a k L)$. Since $c k L=c k H \otimes_{k H} k L$, the same arguments as in the case $a k L$ show that $X \in \chi(c k L)=\chi(b k L)=\chi(a k L)$ if and only if $c k L / c k L I$ is not $k A_{X}$-torsion. Let $X \in \chi(c k L)=\chi(b k L)=\chi(a k L)$ then $c k L / c k L I$ is not $k A_{X^{-}}$-torsion, and it follows from Lemma 3.3 that $b k L / b k L I$ is not $k A_{X^{-}}$ torsion because $c k L \leqslant b k l L$. Suppose now that $b k L / b k L I$ is not $k A_{X}$-torsion then it follows from Lemma 3.3 that $a k L / a k L I$ is not $k A_{X}$-torsion because $b k L \leqslant a k l$ and hence $X \in \chi(c k L)=\chi(b k L)=\chi(a k L)$.

Step 2. Consider now the general case. By step 1, we can chose the element $0 \neq a \in W$ such that the assertion holds for any element $0 \neq b \in$ $a k L$. Besides, as it was mention in the beginning of the proof, $a k N$ satisfies the conditions (i)-(iv) of Lemma 3.2 and the conditions (ii), (iii) of Lemma 3.3 and these conditions hold for any cyclic submodule $0 \neq b k N \leqslant a k N$.

Let $X \in \chi(b k L)$ and suppose that the quotient module $b k L / b k L I$ is $k A_{X}$-torsion. By Lemma 3.3(iii), there is a $k L$-submodule $0 \neq c k L \leqslant a k L$ such that $c k L / c k L I$ has a finite series each of whose quotient is isomorphic to some section of $b k L / b k L I$ considered as $k A$-modules. Therefore, the quotient module $c k L / c k L I$ is $k A_{X}$-torsion. On the other hand, by Lemma 3.2(iii), 
$X \in \chi(c k L)$ and by, step 1 , the quotient module $c k L / c k L I$ is not $k A_{X^{-}}$ torsion. Thus, a contradiction is obtained and hence the quotient module $b k L / b k L I$ is not $k A_{X}$-torsion.

Suppose now that the quotient module $b k L / b k L I$ is not $k A_{X}$-torsion. By Lemma 3.3(ii), $b k L / b k L I$ has a finite series each of whose quotient is isomorphic to some section of $a k L / a k L I$ considered as $k A$-modules and hence the quotient module $a k L / a k L I$ is not $k A_{X}$-torsion. Then, by step $1, X \in$ $\chi(a k L)$ and, by Lemma 3.2(iii), $X \in \chi(b k L)$.

\section{A Finite Set of Commutative Invariants for Modules over Group Rings of Nilpotent Min- imax Groups}

Let $R$ be a commutative Noetherian ring and let $I$ be an ideal of $R$. Let $\mu_{R}(I)$ be the set of prime ideals of $R$ minimal over $I$, by [1, Chap. II, $\S 4$, Corollary 3], the set $\mu_{R}(I)$ is finite.

Let $P$ be a prime ideal of a commutative ring $R$ and let $R_{P}$ be the localization of $R$ at the ideal $P$. Let $M$ be an $R$-module, the support $S u p p_{R} M$ of the module $M$ consists of all prime ideals $P$ of $R$ such that $M_{P}=M \otimes_{R} R_{P} \neq 0$ (see [1, Chap. II, §4]). By [1, Chap. IV, §1, Theorem 2], if $R$ and $M$ are Noetherian then the set $\mu_{R}(M)$ of minimal elements of $\operatorname{Supp}_{R} M$ coincides with $\mu_{R}\left(A n n_{R}(M)\right)$, where $\mu_{R}\left(A n n_{R}(M)\right)$ is the set of prime ideals of $R$ which are minimal over $A n n_{R} M$. Thus, we have

$$
\mu_{R}(M)=\mu_{R}\left(\operatorname{Ann}_{R}(M)\right) .
$$

Lemma 4.1. Let $A$ be a finitely generated abelian group, let $k$ be a field and let $I$ be an ideal of $k A$. Then for any subgroup $B \leq A$ of finite index in $A$ the following assertions hold:

(i) for any $Q \in \mu_{k B}(I \cap k B)$ there is a prime ideal $P$ of $k A$ such that $I \leq P, Q=P \cap k B$ and any such prime $P$ is necessarily in $\mu_{k A}(I)$;

(ii) if $P \in \mu_{k A}(I)$ then $P \cap k B \notin \mu_{k B}(I \cap k B)$ if and only if there is $S \in \mu_{k A}(I)$ such that $S \cap k B<P \cap k B$;

(iii) if $J$ is an ideal of $k A$ such that $\mu_{k A}(I)=\mu_{k A}(J)$ then $\mu_{k B}(I \cap k B)=$ $\mu_{k B}(J \cap k B)$; 
(iv) if $M$ and $M_{1}$ are finitely generated $k A$-modules such that $\mu_{k A}(M)=$ $\mu_{k A}\left(M_{1}\right)$ then $\mu_{k B}(M)=\mu_{k B}\left(M_{1}\right)$.

Proof. (i) Evidently, the quotient ring $k A / I$ is integer over $(k B+I) / I \cong$ $k B /(k B \cap I)$. Then it follows from [26, Ch.V, $\S 2$, Theorem 3] that there is a prime ideal $P \leq k A$ such that $I \leq P$ and $P \cap k B=Q$. If $P \notin \mu_{k A}(I)$ then there is an ideal $P^{\prime} \in \mu_{k A}(I)$ such that $I \leq P^{\prime}<P$ and hence $I \cap k B \leq$ $P^{\prime} \cap k B \leq P \cap k B=Q$ and, as $Q \in \mu_{k B}(I \cap k B)$, we can conclude that $I \cap k B \leq P^{\prime} \cap k B=P \cap k B=Q$. Since $I \leq P^{\prime}<P$, it contradicts Complement 1 to [26, Ch.V, §2, Theorem 3]. Thus, $P \in \mu_{k A}(I)$.

(ii) Let $P \in \mu_{k A}(I)$. Suppose that $P \cap k B \notin \mu_{k B}(I \cap k B)$ then there is a prime ideal $Q \in \mu_{k B}(I \cap k B)$ such that $Q<P \cap k B$. By (i), there is $S \in \mu_{k A}(I)$ such that $Q=S \cap k B$ and hence $S \cap k B<P \cap k B$. Suppose now that there is $S \in \mu_{k A}(I)$ such that $S \cap k B<P \cap k B$. Then $I \cap k B \leq$ $S \cap k B<P \cap k B$ and hence $P \cap k B \notin \mu_{k B}(I \cap k B)$.

(iii) Let $Q \in \mu_{k B}(I \cap k B)$. By (i), there is $P \in \mu_{k A}(I)$ such that $Q=$ $P \cap k B$. Then, by (ii), there are no $S \in \mu_{k A}(I)$ such that $S \cap k B<P \cap k B$. Since $\mu_{k A}(I)=\mu_{k A}(J)$, we can conclude that there is $P \in \mu_{k A}(J)$ such that $Q=P \cap k B$ and there are no $S \in \mu_{k A}(J)$ such that $S \cap k B<P \cap k B$. Then it follows from (ii) that $Q \in \mu_{k B}(J \cap k B)$. The same arguments show that if $Q \in \mu_{k B}(J \cap k B)$ then $Q \in \mu_{k B}(I \cap k B)$ and the assertion follows.

(iv) It follows from (13) that $\mu_{k A}\left(A n n_{k A}(M)\right)=\mu_{k A}(M)=\mu_{k A}\left(M_{1}\right)=$ $\mu_{k A}\left(A n n_{k A}\left(M_{1}\right)\right)$. Then, as $\mu_{k A}\left(A n n_{k A}(M)\right)=\mu_{k A}\left(A n n_{k A}\left(M_{1}\right)\right)$, it follows from (iii) that $\mu_{k B}\left(A n n_{k A}(M) \cap k B\right)=\mu_{k B}\left(A n n_{k A}\left(M_{1}\right) \cap k B\right)$. By (13), we have $\mu_{k B}(M)=\mu_{k B}\left(A n n_{k B}(M)\right)=\mu_{k B}\left(A n n_{k A}(M) \cap k B\right)$ and $\mu_{k B}\left(M_{1}\right)=$ $\mu_{k B}\left(A n n_{k B}\left(M_{1}\right)\right)=\mu_{k B}\left(A n n_{k A}\left(M_{1}\right) \cap k B\right)$. Then, as $\mu_{k B}\left(A n n_{k A}(M) \cap\right.$ $k B)=\mu_{k B}\left(A n n_{k A}\left(M_{1}\right) \cap k B\right)$, we can conclude that $\mu_{k B}(M)=\mu_{k B}\left(M_{1}\right)$.

Let $G$ be a group and let $K$ be a normal subgroup of $G$. Let $L$ be a subgroup of $G$ such that $K \leqslant L \leqslant G$ and the quotient group $L / K$ is finitely generated free abelian. Let $k$ be a field and let $I$ be a $G$-large ideal of the group ring $k K$. Then the quotient group $K / I^{\dagger}$ is finite and, as the quotient group $L / K$ is finitely generated free abelian, it follows from Lemma 2.2(i) that $L / I^{\dagger}$ has a characteristic central torsion-free subgroup $A$ of finite index. Since the quotient group $L / K$ is finitely generated free abelian, the subgroup $A$ is finitely generated free abelian. By [25, Theorem 3.7], the group ring $k A$ is a Noetherian. Let $W$ be a finitely generated $R L$-module then $W / W I$ is a finitely generated $k A$-module and hence $W / W I$ is a Noetherian $k A$-module. So, the finite set $\mu_{k A}(W / W I)=\mu_{k A}\left(A n n_{k A}(W / W I)\right)$ is defined. 
If $P$ is a prime ideal of the group ring $k A$ then the dimension $d(P)$ of $P$ is the Krull dimension of the quotient ring $k A / P$. It is well known that the dimension $d(P)$ coincides with $r(X)$, where $X$ is a maximal subgroup of $A$ such that $k X \cap P=0$. Thus, the dimension of any prime ideal of $k A$ is bounded with $r(A)$ and $d(\mu)$ will denote the maximal dimension of ideals from $\mu$, where $\mu$ is a set of prime ideals of $k A$ (if $\mu=\emptyset$ we put $d(\mu)=-1$ ).

Lemma 4.2. Let $L$ be a minimax torsion-free nilpotent group and let $K$ be a normal subgroup of $L$ such that the quotient group $L / K$ is free abelian. Let $T$ be a subgroup of finite index in $L$ such that $K \leqslant T$. Let $k$ be a field and let $W$ be a cyclic $k L$-module which is $k K$-torsion-free. Let $I$ be an L-large ideal of $k K$ such that $W \neq W I$. Let $A$ be a central torsion-free subgroup of finite index in $L / I^{\dagger}$ and let $B$ be a subgroup of finite index in $A \cap\left(T / I^{\dagger}\right)$. Then:

(i) $\operatorname{Supp}_{k B} W_{1} / W_{1} I \subseteq S \operatorname{Supp}_{k B} W / W I$ for any cyclic $k T$-submodule $W_{1} \leqslant$ $W$;

(ii) for any cyclic $k L$-submodule $W_{1}$ of $W$ and any $P \in \mu_{k A}\left(W_{1} / W_{1} I\right)$ there is $Q \in \mu_{k A}(W / W I)$ such that $Q \leqslant P$;

(iii) $\mu_{k A}\left(W_{2} / W_{2} I\right) \cap \mu_{k A}(W / W I) \subseteq \mu_{k A}\left(W_{1} / W_{1} I\right) \cap \mu_{k A}(W / W I)$ for any cyclic $k L$-submodules $W_{1}$ and $W_{2}$ of $W$ such that $W_{2} \leqslant W_{1}$;

(iv) there exists a cyclic $k L$-submodule $0 \neq V \leqslant W$ such that $\mu_{k B}\left(V_{1} / V_{1} I\right)=$ $\mu_{k B}(V / V I)$ for any cyclic $k L$-submodule $0 \neq V_{1} \leqslant V$ and any subgroup $B \leq A$ of finite index in $A$.

Proof. (i). Since $|L: T|<\infty$, we see that $a k L$ is a finitely generated $k T$ module. If the group $L$ is finitely generated, the assertion is proved in [19, Lemma 5(i)].

Consider now the general case. Suppose that $W=a k L$ and $W_{1}=b k T$, where $b \in a k L$. By [18, Lemma 2.2.5], there is a finitely generated subgroup $H \leqslant L$ such that $W=a k H \otimes_{k H} k L$. Evidently, taking the subgroup $H$ bigger if it is necessary, we can assume that $b \in a k H$ and $L=I^{\dagger}(K \cap$ $H)$. Therefore, $W_{1}=b k(H \cap T) \otimes_{k(H \cap T)} k T$ and, as $L=I^{\dagger}(K \cap H)$, we have $L=I^{\dagger} H$ and $T=I^{\dagger}(H \cap T)$. Then it follows from [18, Lemma 2.2.6] that there are $k H$-module isomorphism $a k L / a k L I \simeq a k H / a k H I^{\prime}$ and $k(H \cap T)$-module isomorphism $b k T / b k T I \simeq b k(H \cap T) / b k(H \cap T) I^{\prime}$, where $I^{\prime}=k H \cap I$ and these isomorphisms induce $k B$-module isomorphisms. So, 
the assertion follows from the considered above case, where the group $L$ is finitely generated.

(ii). We apply (i) in the case where $T=L$ and $A=B$ then $S u p p_{k A} W_{1} / W_{1} I \subseteq$ $\operatorname{Supp}_{k A} W / W I$ and hence $\mu_{k A}\left(W_{1} / W_{1} I\right) \subseteq S u p p_{k A} W_{1} / W_{1} I \subseteq S u p p_{k A} W / W I$. Then the assertion follows from the definition of $\mu_{k A}(W / W I)$.

(iii). By (ii), for any $P \in \mu_{k A}\left(W_{2} / W_{2} I\right) \cap \mu_{k A}(W / W I)$ there is $Q \in$ $\mu_{k A}\left(W_{1} / W_{1} I\right)$ such that $Q \leqslant P$ and, also by (ii), there is $P_{1} \in \mu_{k A}(W / W I)$ such that $P_{1} \leqslant Q \leqslant P$. However, $P \in \mu_{k A}(W / W I)$ and it follows from the minimality of $P$ that $P_{1}=Q=P$. Thus, $P=Q \in \mu_{k A}\left(W_{1} / W_{1} I\right)$ and hence $P \in \mu_{k A}\left(W_{1} / W_{1} I\right) \cap \mu_{k A}(W / W I)$.

(iv)To simplify denotations, we denote $\mu_{k A}$ my $\mu$. By Lemma 4.1(iv), it is sufficient to show that there exists a cyclic $k L$-submodule $0 \neq V \leqslant W$ such that $\mu\left(V_{1} / V_{1} I\right)=\mu(V / V I)$ for any cyclic $k L$-submodule $0 \neq V_{1} \leqslant V$.

Suppose that the assertion is not true then there is a descending chain $\left\{W_{n} \mid n \in \mathbb{N}\right\}$ of cyclic submodules of $W$ such that

$$
\mu\left(W_{n} / W_{n} I\right) \neq \mu\left(W_{n+1} / W_{n+1} I\right)
$$

for each $n \in \mathbb{N}$.

Step 1. Note at first that $\mu\left(W_{t} / W_{t} I\right) \neq \mu\left(W_{n} / W_{n} I\right)$ if $t \neq n$. Suppose that there are $t>n$ such that $\mu\left(W_{t} / W_{t}\right)=\mu\left(W_{n} / W_{n} I\right)$, we can assume that $t$ and $n$ are nearest numbers with this property. Then it follows from the definition of the chain $\left\{W_{n} \mid n \in \mathbb{N}\right\}$ that $t>n+1>n$ and $\mu\left(W_{t} / W_{t} I\right) \neq$ $\mu\left(W_{n+1} / W_{n+1} I\right) \neq \mu\left(W_{n} / W_{n} I\right)$. Since $W_{t} \leqslant W_{n+1} \leqslant W_{n}$, it follows from (iii) that $\mu\left(W_{t} / W_{t} I\right) \cap \mu\left(W_{n} / W_{n} I\right) \subseteq \mu\left(W_{n+1} / W_{n+1} I\right) \cap \mu\left(W_{n} / W_{n} I\right)$ and, as $\mu\left(W_{t} / W_{t} I\right)=\mu\left(W_{n} / W_{n} I\right)$, we have $\mu\left(W_{n} / W_{n} I\right) \subseteq \mu\left(W_{n+1} / W_{n+1} I\right) \cap$ $\mu\left(W_{n} / W_{n} I\right)$. Therefore, $\mu\left(W_{n} / W_{n} I\right) \subseteq \mu\left(W_{n+1} / W_{n+1} I\right)$. By (ii), for any $P \in \mu\left(W_{n+1} / W_{n+1} I\right)$ there is $Q \in \mu\left(W_{n} / W_{n} I\right)$ such that $Q \leqslant P$ and, as $\mu\left(W_{n} / W_{n} I\right) \subseteq \mu\left(W_{n+1} / W_{n+1} I\right)$, it follows from the minimality of $P$ that $Q=$ $P$. It implies that $\mu\left(W_{n} / W_{n} I\right) \supseteq \mu\left(W_{n+1} / W_{n+1} I\right)$ and hence $\mu\left(W_{n} / W_{n} I\right)=$ $\mu\left(W_{n+1} / W_{n+1} I\right)$ but this contradicts the definition of the chain $\left\{W_{n} \mid n \in N\right\}$. Thus, $\mu\left(W_{t} / W_{t} I\right) \neq \mu\left(W_{n} / W_{n} I\right)$ if $t \neq n$.

Step 2. It follows from (iii) that $\mu\left(W_{t} / W_{t} I\right) \cap \mu\left(W_{1} / W_{1} I\right) \subseteq \mu\left(W_{m} / W_{m} I\right) \cap$ $\mu\left(W_{1} / W_{1} I\right)$ for any $t>m>1$. Then, as the set $\mu\left(W_{1} / W_{1} I\right)$ is finite, there is $m \in \mathbb{N}$ such that

$$
\mu\left(W_{t} / W_{t} I\right) \cap \mu\left(W_{1} / W_{1} I\right)=\mu\left(W_{m} / W_{m} I\right) \cap \mu\left(W_{1} / W_{1} I\right)
$$

for all $t>m$. We put $W_{m}=U_{2}$ and $W_{1}=U_{1}$. The same arguments show that there is $m \in \mathbb{N}$ such that 


$$
\mu\left(W_{t} / W_{t} I\right) \cap \mu\left(U_{2} / U_{2} I\right)=\mu\left(W_{m} / W_{m} I\right) \cap \mu\left(U_{2} / U_{2} I\right)
$$

for all $t>m$. We put $W_{m}=U_{3}$. Continuing this process we obtain a descending chain $\left\{U_{n} \mid n \in \mathbb{N}\right\} \subseteq\left\{W_{n} \mid n \in \mathbb{N}\right\}$ such that

$$
\mu\left(U_{t} / U_{t} I\right) \cap \mu\left(U_{n} / U_{n} I\right)=\mu\left(U_{m} / U_{m} I\right) \cap \mu\left(U_{n} / U_{n} I\right)
$$

for all $t, m, n \in \mathbb{N}$ such that $t \geqslant m \geqslant n$. Since $\mu\left(W_{t} / W_{t} I\right) \neq \mu\left(W_{n} / W_{n} I\right)$ if $t \neq n$, we see that

$$
\mu\left(U_{t} / U_{t} I\right) \neq \mu\left(U_{n} / U_{n} I\right)
$$

if $t \neq n$.

Show now that if

$$
d\left(\mu\left(U_{n+1} / U_{n+1} I\right) \backslash \mu\left(U_{n} / U_{n} I\right)\right) \geqslant 0
$$

(i.e. $\left.\mu\left(U_{n+1} / U_{n+1} I\right) \backslash \mu\left(U_{n} / U_{n} I\right) \neq \emptyset\right)$ then

$$
d\left(\mu\left(U_{n+1} / U_{n+1} I\right) \backslash \mu\left(U_{n} / U_{n} I\right)\right)<d\left(\mu\left(U_{n} / U_{n} I\right) \backslash \mu\left(U_{n-1} / U_{n-1} I\right)\right)
$$

for any $n \geqslant 2$. By (ii), for any $P \in \mu\left(U_{n+1} / U_{n+1} I\right) \backslash \mu\left(U_{n} / U_{n} I\right)$ there is $Q \in \mu\left(U_{n} / U_{n} I\right)$ such that $Q \leqslant P$ and, as $P \notin \mu\left(U_{n} / U_{n} I\right)$, we have $Q<P$. By (14), $\mu\left(U_{n+1} / U_{n+1} I\right) \cap \mu\left(U_{n-1} / U_{n-1} I\right)=\mu\left(U_{n} / U_{n} I\right) \cap \mu\left(U_{n-1} / U_{n-1} I\right)$ and hence if $Q \in \mu\left(U_{n-1} / U_{n-1} I\right)$ then $Q \in \mu\left(U_{n+1} / U_{n+1} I\right)$ but $P \in \mu\left(U_{n+1} / U_{n+1} I\right)$ and it contradicts $Q<P$. Thus, $Q \notin \mu\left(U_{n-1} / U_{n-1} I\right)$ and hence $Q \in$ $\mu\left(U_{n} / U_{n} I\right) \backslash \mu\left(U_{n-1} / U_{n-1} I\right)$. So, for any $P \in \mu\left(U_{n+1} / U_{n+1} I\right) \backslash \mu\left(U_{n} / U_{n} I\right)$ there is $Q \in \mu\left(U_{n} / U_{n} I\right) \backslash \mu\left(U_{n-1} / U_{n-1} I\right)$ such that $Q<P$ and hence $\left.d(Q)\right\rangle$ $d(P)$ and the relation (16) follows.

The relation (16) easily implies that there is $m \in \mathbb{N}$ such that

$$
d\left(\mu\left(U_{n+1} / U_{n+1} I\right) \backslash \mu\left(U_{n} / U_{n} I\right)\right)=-1
$$

(i.e. $\left.\mu\left(U_{n+1} / U_{n+1} I\right) \backslash \mu\left(U_{n} / U_{n} I\right)=\emptyset\right)$ for all $n \geqslant m$. Therefore, $\mu\left(U_{n+1} / U_{n+1} I\right) \subseteq$ $\left.\mu\left(U_{n} / U_{n} I\right)\right)$. Then it follows from (14) that for any $t>n+1$ we have $\mu\left(U_{t} / U_{t} I\right) \cap \mu\left(U_{n} / U_{n} I\right)=\mu\left(U_{n+1} / U_{n+1} I\right)$. Therefore, $\mu\left(U_{n+1} / U_{n+1} I\right) \subseteq$ $\mu\left(U_{t} / U_{t} I\right)$. Then, as $t>n+1$, it easily follows from (ii), that $\mu\left(U_{n+1} / U_{n+1} I\right)=$ $\mu\left(U_{t} / U_{t} I\right)$ but it contradicts (15). 
Lemma 4.3. Let $N$ be a minimax torsion-free nilpotent group and let $K$ be a normal subgroup of $N$ such that the quotient group $N / K$ is torsion-free abelian. Let $L$ be a dense subgroup of $N$ such that $K \leqslant L$ and the quotient group $L / K$ is free abelian. Let $k$ be a field and let $W$ be a $k N$-module which is $k K$-torsion-free. Let $I$ be an $N$-large ideal of $k K$ such that $W \neq W I$ and $A$ be a central subgroup of finite index in $L / I^{\dagger}$. Then there exists a cyclic $k N$-submodule $0 \neq U \leqslant W$ such that $\mu_{k B}(b k L / b k L I)=\mu_{k B}(d k L / d k L I)$ for any elements $0 \neq b, d \in U$ and any subgroup $B \leq A$ of finite index in $A$.

Proof. By Lemma 4.1(iv), it is sufficient to show that there exists a cyclic $k N$-submodule $0 \neq U \leqslant W$ such that $\mu_{k A}(b k L / b k L I)=\mu_{k A}(d k L / d k L I)$ for any elements $0 \neq b, d \in U$.

By Lemma 3.3(ii), there is a cyclic $k L$-submodule $0 \neq V \leqslant W$ such that for any element $0 \neq a \in V$ and any element $0 \neq b \in a k N$ the $k A$-module $b k L / b k L I$ has a finite series each of whose quotient is isomorphic to some section of the $k A$-module $a k L / a k L I$. Then it follows from [1, Ch. II, $\S 4$, Proposition 16] that

$$
\operatorname{Supp}_{k A} b k L / b k L I \subseteq \operatorname{Supp}_{k A} a k L / a k L I .
$$

By Lemma 3.3(iii), there is a $k L$-submodule $0 \neq c k L \leqslant a k L$ such that the $k A$-module $c k L / c k L I$ has a finite series each of whose quotient is isomorphic to some section of the $k A$-module $b k L / b k L I$. Then it follows from [1, Ch. II, §4, Proposition 16] that

$$
\operatorname{Supp}_{k A} c k L / c k L I \subseteq \operatorname{Supp}_{k A} b k L / b k L I .
$$

By Lemma 4.2(iv), we also can assume that $\mu_{k A}\left(V_{1} / V_{1} I\right)=\mu_{k A}(V / V I)$ for any cyclic $k L$-submodule $0 \neq V_{1} \leqslant V$ and hence

$$
\mu_{k A}(c k L / c k L I)=\mu_{k A}(a k L / a k L I)
$$

If $P \in \mu_{k A}(a k L / a k L)$ and $P \notin \mu_{k A}(b k L / b k L I)$ then it follows from (19) that $P \in \mu_{k A}(c k L / c k L)$ and $P \notin \mu_{k A}(b k L / b k L I)$. Therefore, it follows from (18) that there is $Q \in \mu_{k A}(b k L / b k L I)$ such that $Q<P$. But then (17) implies that $P \notin \mu_{k A}(a k L / a k L)$ and the obtained contradiction shows that $\mu_{k A}(a k L / a k L) \subseteq \mu_{k A}(b k L / b k L I)$

If $P \in \mu_{k A}(b k L / b k L I)$ and $P \notin \mu_{k A}(a k L / a k L)$ then it follows from (17) that there is $Q \in \mu_{k A}(a k L / a k L I)$ such that $Q<P$ and (19) implies that $Q \in \mu_{k A}(c k L / c k L I)$. But then (18) implies that $P \notin \mu_{k A}(b k L / b k L)$ and the 
obtained contradiction shows that $\mu_{k A}(a k L / a k L) \supseteq \mu_{k A}(b k L / b k L I)$. Thus, we can conclude that $\mu_{k A}(a k L / a k L)=\mu_{k A}(b k L / b k L I)$. Put $U=a k N$ then we have $\mu_{k A}(a k L / a k L)=\mu_{k A}(b k L / b k L I)$ for any element $0 \neq b \in U$ and the assertion follows.

We also need some notions introduced by Wilson in [23, Section 3.8] which are based on results of Brookes [2]. Let $A$ be a torsion-free abelian group of finite rank and let $k$ be a field. If $I$ and $J$ are ideals of $k A$ then we write $I \approx J$ if $I \cap k B=J \cap k B$ for some finitely generated dense subgroup $B \leqslant A$. Then $\approx$ is an equivalence relation on the set of all prime ideals of $k A$ and we denote by $[I]$ the class of equivalence containing an ideal $I$. If a group $G$ acts on $A$ then we obtain an action of $G$ on the set of equivalent prime ideals of $k A$ which is given by $[I]^{g}=\left[I^{g}\right]$.

If $B$ is a dense subgroup of $A$ and $P$ is a prime ideal of $k B$ then, as $k A$ is an integer domain over $k B$, it follows from [1, Chap. V, $\S 2$, Theorem 1] that there is a prime ideal $Q$ of $k A$ such that $Q \cap k B=P$ and we put $[P]_{k A}=[Q]$. If $\mu$ is a set of prime ideals of $k B$ then we put $[\mu]_{k A}=\left\{[P]_{k A} \mid P \in \mu\right\}$.

Lemma 4.4. Let $A$ be a torsion-free abelian group of finite rank, let $B$ be a dense subgroup of $A$, let $k$ be a field and let $I$ be an ideal of $k B$. Then:

(i) there is a finitely generated dense subgroup $C \leqslant B$ such that for any subgroup $X$ of finite index in $C$ the mapping $\mu_{k C}(I \cap k C) \rightarrow \mu_{k X}(I \cap k X)$ given by $P \mapsto P \cap k X$ is bijective;

(ii) for any finitely generated dense subgroup $C \leqslant B$ which meets (i) we have $\left|\left[\mu_{k C}(I \cap k C)\right]_{k A}\right|=\left|\mu_{k C}(I \cap k C)\right|<\infty$;

(iii) for any finitely generated dense subgroups $C, D \leqslant B$ which meet (i) we have $\left[\mu_{k C}(I \cap k C)\right]_{k A}=\left[\mu_{k D}(I \cap k D)\right]_{k A}$;

Proof. (i) It follows from [2, Lemma 2.1] that there is a finitely generated dense subgroup $C \leqslant B$ such that for any subgroup $X$ of finite index in $C$ if $P \in \mu_{k X}(I \cap k C)$ then $\varphi_{X}: P \mapsto P \cap k X$ maps $\mu_{k C}(I \cap k C)$ into $\mu_{k X}(I \cap k X)$. It follows from Lemma 4.1(i) that the mapping $\varphi_{X}$ is surjective. If $\varphi_{X}$ is not injective for some subgroup $X$ of finite index in $C$, then $\left|\mu_{k X}(I \cap k X)\right|<$ $\left|\mu_{k C}(I \cap k C)\right|$ and we can replace the subgroup $C$ by its subgroup $X$ of finite index with minimal possible $\left|\mu_{k X}(I \cap k X)\right|$.

(ii) Let $[P],[Q] \in\left[\mu_{k C}(I \cap k C)\right]_{k A}$ then $P \cap k C$ and $Q \cap k C$ are in $\mu_{k C}(I \cap$ $k C) .[P]=[Q]$ if and only if there is a finitely generated dense subgroup 
$X$ of $A$ such that $P \cap k X=Q \cap k X$. Evidently, we can assume that $X \leqslant$ $C$ and, as the mapping $\mu_{k C}(I \cap k C) \rightarrow \mu_{k X}(I \cap k X)$ given by $P \mapsto P \cap$ $k X$ is bijective, we can conclude that $P \cap k C=Q \cap k C$. Therefore, for any $U, V \in \mu_{k C}(I \cap k C)$ if $U \neq V$ then $[U]_{k A} \neq[V]_{k A}$ and it implies that $\left|\left[\mu_{k C}(I \cap k C)\right]_{k A}\right|=\left|\mu_{k C}(I \cap k C)\right|$. As the ring $k C$ is Noetherian, it follows from [1, Chap. IV, §1, Theorem 2] that $\left|\mu_{k C}(I \cap k C)\right|<\infty$.

(iii) It is easy to note that the subgroup $X=D \cap C \leq A$ meets (i) and hence it is sufficient to show that $\left[\mu_{k C}(I \cap k C)\right]_{k A}=\left[\mu_{k X}(I \cap k X)\right]_{k A}=$ $\left[\mu_{k D}(I \cap k D)\right]_{k A}$. Thus, we can consider only the case, where $X=D \leqslant C$. If $[P] \in\left[\mu_{k C}(I \cap k C)\right]_{k A}$ then, by the definition of $\left[\mu_{k C}(I \cap k C)\right]_{k A}$, we can assume that $P \cap k C \in \mu_{k C}(I \cap k C)$. As by (i), $\varphi_{X}: P \mapsto P \cap k X$ maps $\mu_{k C}(I \cap k C)$ into $\mu_{k X}(I \cap k X)$ we have $P \cap k X \in \mu_{k X}(I \cap k X)$ and hence $[P] \in\left[\mu_{k X}(I \cap k X)\right]_{k A}$. On the other hand, if $[P] \in\left[\mu_{k X}(I \cap k X)\right]_{k A}$ then, by the definition of $\left[\mu_{k X}(I \cap k X)\right]_{k A}$, we can assume that $P \cap k X \in \mu_{k X}(I \cap k X)$. By Lemma 4.1(i), there is $Q \in \mu_{k C}(I \cap k C)$ such that $Q \cap k X=P \cap k X$. Let $[S]=[Q]_{k A}$ then $[S] \in\left[\mu_{k C}(I \cap k C)\right]_{k A}$ and $S \cap k X=P \cap k X$. Therefore, $[P]=[S] \in\left[\mu_{k C}(I \cap k C)\right]_{k A}$.

Let $G$ be a group and let $K$ be a normal subgroup of $G$. Let $N$ be a nilpotent subgroup of $G$ such that $K \leqslant N \leqslant G$ and the quotient group $N / K$ is torsion-free abelian of finite rank. Let $k$ be a field and let $I$ be a $G$ large ideal of $k K$. Then, as the quotient group $K / I^{\dagger}$ is finite and the quotient group $N / K$ is torsion-free abelian of finite rank, it follows from Lemma 2.2(i) that $N / I^{\dagger}$ has a characteristic central torsion-free subgroup $A$ of finite index.

Let $L$ be a dense subgroups of $N$ such that $K \leqslant L$ and the quotient groups $L / K$ is finitely generated. Then $A \cap L / I^{\dagger}$ is a dense central finitely generated torsion-free subgroup of $A$.

Let $W$ be a $k N$-module which is $k K$-torsion-free and let $a k L$ be a cyclic $k L$-module generated by an element $0 \neq a \in W$. By Lemma 4.4(i), there is a finitely generated dense subgroup $A_{L} \leqslant A \cap L / I^{\dagger}$ such that for any subgroup $X$ of finite index in $A_{L}$ the mapping $\mu_{k A_{L}}\left(A n n_{k A_{L}}(a k L / a k L I)\right) \rightarrow$ $\mu_{k X}\left(A n n_{k X}(a k L / a k L I)\right)$ given by $P \mapsto P \cap k X$ is bijective.

As $A_{L}$ is a finitely generated subgroup of finite index in $A \cap L / I^{\dagger}$, we can conclude that $A_{L}$ is a dense finitely generated subgroup of $A$ and $a k L / a k L I$ is a finitely generated $k A_{L}$-module. Then it follows from [25, Theorem 3.7] that the domain $k A_{L}$ is Noetherian and akL/akLI is a Noetherian $k A_{L^{-}}$ module. Thus, the sets $S_{u p p_{k A_{L}}}(a k L / a k L I)$ and $\mu_{k A_{L}}(a k L / a k L I)$ are well 
defined. Then according to (13) we have

$$
\mu_{k A_{L}}(a k L / a k L I)=\mu_{k A_{L}}\left(A n n_{k A_{L}}(a k L / a k L I)\right)
$$

Thus, the set $\mu_{k A_{L}}(a k L / a k L I)$ is defined for any $0 \neq a \in W$ and we put $\left[\mu_{k A_{L}}(a k L / a k L I)\right]_{k A}=\left\{[P]_{k A} \mid P \in \mu_{k A_{L}}(a k L / a k L I)\right\}$. It follows from (20) that

$$
\left[\mu_{k A_{L}}(a k L / a k L I)\right]_{k A}=\left[\mu_{k A_{L}}\left(A n n_{k A_{L}}(a k L / a k L I)\right)\right]_{k A}
$$

By (21) and according to Lemma 4.4(ii),(iii), the set $\left[\mu_{k A_{L}}(a k L / a k L I)\right]_{k A}=$ $\left\{[P]_{k A} \mid P \in \mu_{k A_{L}}\left(A n n_{k A_{L}}\left(a_{L}\right)\right)\right\}$ is finite and does not depend on the choice of the subgroup $A_{L}$ which meets the conditions of Lemma 4.4(i). Everywhere below in the definition of the set

$$
\left[\mu_{k A_{L}}(a k L / a k L I)\right]_{k A}=\left\{[P]_{k A} \mid P \in \mu_{k A_{L}}(a k L / a k L I)\right\}
$$

we assume that the subgroup $A_{L}$ meets the conditions of Lemma 4.4(i),i.e. for any subgroup $X$ of finite index in $A_{L}$ the mapping $\mu_{k A_{L}}\left(A n n_{k A_{L}}(a k L / a k L I)\right) \rightarrow$ $\mu_{k X}\left(A n n_{k X}(a k L / a k L I)\right)$ given by $P \mapsto P \cap k X$ is bijective. .

Proposition 4.1. Let $N$ be a minimax torsion-free nilpotent group and let $K$ be a normal subgroup of $N$ such that the quotient group $N / K$ is torsion-free abelian. Let $k$ be a field such that chark $=0$ and let $W$ be a $k N$-module which is $k K$-torsion-free. Let $I$ be an $N$-large ideal of $k K$ and $A$ be a torsion-free characteristic central subgroup of finite index in $N / I^{\dagger}$. Then there exists a cyclic $k N$-submodule $0 \neq V \leqslant W$ such that $\left[\mu_{k A_{L}}(a k L / a k L I)\right]_{k A}=\left[\mu_{k A_{M}}(b k M / b k M I)\right]_{k A}$ for any elements $0 \neq a, b \in V$ and any dense subgroups $L, M \leqslant N$ such that $K \leqslant L \cap M$ and the quotient groups $L / K$ and $M / K$ are finitely generated.

Proof. At first we fix a dense subgroup $L \leqslant N$ such that $K \leqslant L$ and the quotient group $L / K$ is finitely generated. By Lemma 4.3, we can choose an element $0 \neq a \in W$ such that $\mu_{k B_{L}}(a k L / a k L I)=\mu_{k B_{L}}(b k L / b k L I)$ for any $0 \neq b \in a k N$ and any subgroup $B_{L}$ of finite index in $A_{L}$. Put $V=a k N$ then it is sufficient to show that $\left[\mu_{k A_{L}}(b k L / b k L I)\right]_{k A}=\left[\mu_{k A_{M}}(b k M / b k M I)\right]_{k A}$ for any $0 \neq b \in a k N$ and any dense subgroup $M \leqslant N$ such that $K \leqslant M$ and the quotient group $M / K$ is finitely generated.

It is easy to note that $T=L \cap M$ is a subgroup of finite index in $L$ and $M$. It easily implies that we can choose a finitely generated dense subgroup $A_{T} \leqslant A \cap\left(T / I^{\dagger}\right)$ such that $A_{T} \leqslant A_{L} \cap A_{M}$. Then it is sufficient to show 
that $\left[\mu_{k A_{L}}(b k L / b k L I)\right]_{k A}=\left[\mu_{k A_{T}}(b k T / b k T I)\right]_{k A}=\left[\mu_{k A_{M}}(b k M / b k M I)\right]_{k A}$. In fact it is sufficient to prove the identity

$$
\left[\mu_{k A_{L}}(b k L / b k L I)\right]_{k A}=\left[\mu_{k A_{T}}(b k T / b k T I)\right]_{k A}
$$

because the identity $\left[\mu_{k A_{T}}(b k T / b k T I)\right]_{k A}=\left[\mu_{k A_{M}}(b k M / b k M I)\right]_{k A}$ is analogous.

By Lemma 4.4(iii), $\left[\mu_{k A_{L}}(b k L / b k L I)\right]_{k A}=\left[\mu_{k A_{T}}(b k L / b k L I)\right]_{k A}$ and hence it is sufficient to show that $\left[\mu_{k A_{T}}(b k T / b k T I)\right]_{k A}=\left[\mu_{k A_{T}}(b k L / b k L I)\right]_{k A}$. Since $T \leqslant L$, we have $b k T \leqslant b k L$ and it follows from Lemma 4.2(i) that

$$
\operatorname{Supp}_{k A_{T}} b k T / b k T I \subseteq S u p p_{k A_{T}} b k L / b k L I .
$$

On the other hand, as $T \leqslant L$, we see that $b k L=\sum_{i=1}^{n} b k T g_{i}$ for some $g_{i} \in L$. Therefore,

$$
b k L / b k L I \cong\left(\sum_{i=1}^{n} b k T g_{i} / b k T g_{i} I\right) / X
$$

where $X$ is a $k A_{T}$-submodule of $\sum_{i=1}^{n} b k T g_{i} / b k T g_{i} I$. As $A_{T}$ is a central subgroup of $N / I^{\dagger}$, we can conclude that $b k T g_{i} / b k T g_{i} I \cong b k T / b k T I$ for all $g_{i} \in L$. Then it follows from (23) and [1, Ch. II, §4, Proposition 16] that

$$
\operatorname{Supp}_{k A_{T}} b k L / b k L I \subseteq S u p p_{k A_{T}} b k T / b k T I .
$$

The relations (22) and (24) imply that $S u p p_{k A_{T}} b k L / b k L I=S u p p_{k A_{T}} b k T / b k T I$ and hence $\mu_{k A_{T}}(b k L / b k L I)=\mu_{k A_{T}}(b k T / b k T I)$. Therefore, $\left[\mu_{k A_{T}}(b k T / b k T I)\right]_{k A}=$ $\left[\mu_{k A_{T}}(b k L / b k L I)\right]_{k A}$.

Corollary 4.1. In the denotations of Proposition 4.1, the cyclic $k N$-submodule $0 \neq V \leqslant W$ defines a finite set

$$
M_{k A}(V / V I)=\left[\mu_{k A_{L}}(a k L / a k L I)\right]_{k A}
$$

of equivalent classes of prime ideals of $k A$ which depends only on the ideal $I$ and the subgroup $A$.

Proof. The finiteness on (25) follows from (21) and Lemma 4.4(ii),(iii). By Proposition 4.1, (25) does not depend on the choice of the subgroup $L$ and the element $0 \neq a \in V$. By Lemma 4.4(iii), (25) does not depend on the choice of the subgroup $A_{L}$ which meets the conditions of Lemma 4.4(i). 
Combining Propositions 3.1, 3.2 and Corollary 4.1 we obtain the following theorem

Theorem 4.1. Let $G$ be a soluble group of finite torsion-free rank $r_{0}(G)<$ $\infty$, let $N$ be a minimax nilpotent torsion-free normal subgroup of $G$ and let $K$ be a $G$-invariant subgroup of $N$ such that the quotient group $N / K$ is torsionfree abelian. Let $L$ be a dense subgroup of $N$ such that $K \leqslant L$ and the quotient group $L / K$ is free abelian and let $\chi$ be a full system of subgroups of $L$ over $K$. Let $k$ be a field of characteristic zero and let $W$ be a $k N$-module which is $k K$-torsion-free. Then there are a cyclic $k N$-submodule $0 \neq V \leqslant W$, a $G$-large ideal $I$ of $k K$ and a central $G$-invariant subgroup $A$ of finite index in $N / I^{\dagger}$ such that for any element $0 \neq b \in V$ we have:

(i) $X \in \chi(b k L)$ if and only if the quotient module bkL/bkLI is not $k A_{X^{-}}$ torsion, where $A_{X}=A \cap X / I^{\dagger}$;

(ii) for any dense subgroup $M \leqslant N$ with $K \leqslant M$ and finitely generated quotient group $M / K$ the finite set $M_{k A}(V / V I)=\left[\mu_{k A_{M}}(b k M / b k M I)\right]_{k A}$ of equivalent classes of prime ideals of $k A$ depends only on the ideal I and the subgroup $A$;

(iii) for any $g \in G$ we have $M_{k A}(V g / V g I)=M_{k A}(V / V I)^{g}=\left\{[P]^{g}=\right.$ $\left.\left[P^{g}\right] \mid[P] \in M_{k A}(V / V I)\right\}$.

Proof. (i) By Propositions 3.2, there are a cyclic $k N$-submodule $0 \neq U \leqslant W$, a finitely generated dense subgroup $H$ of $L$ and a right ideal $J$ of $k(H \cap K)$ such that if $I$ is an $N$-large ideal of $k K$ such that $I \cap k(K \cap H)$ culls $J$ in $k(H \cap K)$ then for any $0 \neq b \in U$ we have $X \in \chi(b k L)$ if and only if the quotient module $b k L / b k L I$ is not $k A_{X}$-torsion, where $A_{X}=A \cap X / I^{\dagger}$ and $A$ is central subgroup of finite index in $N / I^{\dagger}$. By Propositions 3.1, there is a $G$-large ideal $I$ of $k K$ such that $I \cap k(K \cap H)$ culls $J$ in $k(H \cap K)$. As $N \leqslant G$, we can conclude that the ideal $I$ is an $N$-large and hence the assertion (i) holds for the ideal $I$ and the submodule $U$. Since $N, I^{\dagger}$ and $K$ are normal subgroups of $G$ and any abelian-by-finite nilpotent group of finite rank has a characteristic central subgroup of finite index, we can chose the subgroup $A$ such that $A$ is $G$-invariant. Then replacing $W$ by $U$ we can assume that (i) holds for any $0 \neq b \in W$.

(ii) By Corollary 4.1, we can choose a cyclic submodule $0 \neq V \leqslant W$ such that for any $0 \neq b \in V$ and any dense subgroup $M \leqslant N$ with $K \leqslant M$ 
and finitely generated quotient group $M / K$ the finite set $M_{k A}(V / V I)=$ $\left[\mu_{k A_{M}}(b k M / b k M I)\right]_{k A}$ depends only on the ideal $I$ and the subgroup $A$.

(iii) Since the subgroup $A$ and the ideal $I$ are $G$-invariant, we have $M_{k A}(V g / V g I)=\left[\mu_{k A_{L}^{g}}(b g k L / b g k L I)\right]_{k A}$. Then by (21),$M_{k A}(V g / V g I)=$ $\left[\mu_{k A_{L}^{g}}(b g k L / b g k L I)\right]_{k A}=\left[\mu_{k A_{L}^{g}}\left(A n n_{k A_{L}^{g}}(b g k L / b g k L I)\right)\right]_{k A}$. It is not difficult to show that

$$
\left[\mu_{k A_{L}^{g}}\left(A n n_{k A_{L}^{g}}(b g k L / b g k L I)\right)\right]_{k A}=\left[\mu_{k A_{L}}\left(\left(A n n_{k A_{L}}(b k L / b k L I)\right)^{g}\right)\right]_{k A}
$$

and hence $M_{k A}(V g / V g I)=\left[\mu_{k A_{L}}\left(\left(A n n_{k A_{L}}(b k L / b k L I)\right)^{g}\right)\right]_{k A}$.

Thus, $M_{k A}(V g / V g I)=\left[\left(\mu_{k A_{L}}\left(A n n_{k A_{L}}(b k L / b k L I)\right)^{g}\right]_{k A}=\left\{\left[P^{g}\right]_{k A} \mid P \in\right.\right.$ $\left(\mu_{k A_{L}}\left(A_{n n_{k A_{L}}}(b k L / b k L I)\right)\right\}=\left\{[P]_{k A}^{g} \mid P \in\left(\mu_{k A_{L}}\left(A n n_{k A_{L}}(b k L / b k L I)\right)\right\}=\right.$ $\left\{[P]^{g}=\left[P^{g}\right] \mid[P] \in M_{k A}(V / V I)\right\}=M_{k A}(V / V I)^{g}$.

\section{Uniform Stabilized Modules over Minimax Nilpotent Groups}

Let $A$ be an abelian torsion-free group of finite rank acted by a group $G$, let $k$ be a field and let $I$ be an ideal of $k A$. A subgroup $S_{G}(I) \leqslant G$ which consists of all elements $g \in G$ such that $I \cap k B=I^{g} \cap k B$ for some finitely generated dense subgroup $B \leqslant A$ is said to be the standardizer of $I$ in $G$ (see [2,23]). Since the class $[I]$ consists of all ideals $J \leqslant k A$ such that that $I \cap k B=J \cap k B$ for some finitely generated dense subgroup $B \leqslant A$, we see that $[I]^{g}=\left\{J^{g} \mid J \in[I]\right\}$ also forms a class $\left[I^{g}\right]$. So, we have an action of $G$ on the set of classes of ideals of $k A$. It immediately follows from the definition of $S_{G}(I)$ that $S_{G}(I)=S_{G}([I])$, where $S_{G}([I])=\left\{g \in G \mid[I]^{g}=[I]\right\}$ is the stabilizer of $[I]$ in $G$.

Lemma 5.1. Let $R$ be a Noetherian domain, let $A$ be a finitely generated abelian group and let $I$ be an ideal of the group ring $R A$. Let $B$ be a torsionfree subgroup of $A$ such that $R B \cap P \neq 0$ for any $P \in \mu_{R}(I)$. Then $R B \cap I \neq 0$.

Proof. By [25, Theorem 3.7], the ring $R A$ is Noetherian and it follows from [9, Corollary 37.11] that $k B$ is a domain. Then, by [26, Chap. IV, §5, Theorem 5], the ideal $I$ has a presentation $I=\cap_{i=1}^{n} D_{i}$, where $D_{i}$ are primary ideals of $R A$. Let $P_{i}$ be the prime radical of $D_{i}$, then $P_{i}$ is a prime over $I$ and it follows from the definition of $\mu_{R}(I)$ that there is $P \in \mu_{R}(I)$ such that $P_{i} \geqslant P$. So, as $R B \cap P \neq 0$, we can conclude that there is an element 
$0 \neq a_{i} \in R B \cap P_{i} \neq 0$ for each $i$. Then it follows from [26, Chap. III, $\S 9$, Theorem 13] that that there is an integer $m_{i} \in \mathbb{N}$ such that $a_{i}{ }^{m_{i}} \in D_{i}$ and therefore $0 \neq \prod_{i=1}^{n} a_{i}{ }^{m_{i}} \in I \cap R B$.

Lemma 5.2. Let $A$ be an abelian torsion-free group of finite rank acted by a soluble-by-finite group $G$, let $C=S_{0 c_{G}} A$ and let $B$ be a finitely generated dense subgroup of $A$. Let $k$ be a field of characteristic zero and let $J$ be an ideal of $k B$. Let $P_{1}, P_{2}, \ldots, P_{n}$ be prime ideals of $k A$ such that $\mu_{k B}(J)=\left\{P_{i} \cap k B \mid 1 \leqslant i \leqslant n\right\}$ is the set of minimal prime ideals over $J$. If $\left|G: S_{G}\left(\left[P_{i}\right]\right)\right|<\infty$ for all $1 \leqslant i \leqslant n$ then $J \cap k(C \cap B) \neq 0$.

Proof. Since $\left|G: S_{G}\left(\left[P_{i}\right]\right)\right|<\infty$ for all $1 \leqslant i \leqslant n$, we can conclude that $G$ has a normal subgroup $H$ of finite index such that $H=S_{H}\left(\left[P_{i}\right]\right)$ for all $1 \leqslant i \leqslant n$. By Lemma 2.4(i), $\operatorname{Soc}_{H} A=\operatorname{Soc}_{G} A$ and hence there is no harm in assuming that $H=G$. Thus, we may assume that $G=S_{G}\left(\left[P_{i}\right]\right)=S_{G}\left(P_{i}\right)$ for all $1 \leqslant i \leqslant n$.

Suppose that $P_{i}^{\dagger}=\left\{a \in A \mid 1-a \in P_{i}\right\} \neq 1$. As $i s\left(P_{i}^{\dagger}\right)^{g}=i s\left(\left(P_{i}^{g}\right)^{\dagger}\right)$ for all $g \in G$ and $G=S_{G}\left(P_{i}\right)$, we can conclude that $i s\left(P_{i}^{\dagger}\right)^{g}=i s\left(P_{i}^{\dagger}\right)$ for all $g \in G$, i.e. $i s\left(P_{i}^{\dagger}\right)$ is a $G$-invariant subgroup of $A$. Then $i s\left(P_{i}^{\dagger}\right) \cap C \neq 1$ and as $C$ is an isolated subgroup of $A$, we see that $P_{i}^{\dagger} \cap C \neq 1$. It easily implies that $P_{i} \cap k C \neq 0$.

Suppose now that $P_{i}^{\dagger}=\left\{a \in A \mid 1-a \in P_{i}\right\}=1$. Then by [2, Theorem A] (see also [21, Theorem 2.4]), $P_{i}=\left(P_{i} \cap k \Delta_{G}(A)\right) k A$ and hence $P_{i} \cap k C \neq 0$ because, by Lemma $2.3(\mathrm{i}), \Delta_{G}(A) \leqslant \operatorname{Soc}_{G}(A)=C$.

Thus, $P_{i} \cap k C \neq 0$ for all $1 \leqslant i \leqslant n$. Then, as $B$ is a dense subgroup of $A$, it follows from [18, Lemma 2.2.3(ii)] that $P_{i} \cap k(C \cap B) \neq 0$ for all $1 \leqslant i \leqslant n$. Since $\mu_{k B}(J)=\left\{P_{i} \cap k B \mid 1 \leqslant i \leqslant n\right\}$ and $P_{i} \cap k(C \cap B) \neq 0$ for all $1 \leqslant i \leqslant n$, it follows from Lemma 5.1 that $J \cap k(C \cap B) \neq 0$.

Let $R$ be a ring and let $M, X$ and $Y$ be $B$-modules. The modules $X$ and $Y$ are separated in $M$ if $X$ and $Y$ do not have non-zero isomorphic sections which are isomorphic to a submodule of $M$. Let $k$ be a field, $G$ be a group and $A$ be a normal subgroup of $G$. Let $M$ and $W$ be $k A$-modules. The subgroup $\operatorname{Sep}_{(G, A)}(M, W) \leqslant G$ generated by all elements $g \in G$ such that $k A$-modules $W$ and $W g$ are not separated in $M$ is said to be the separator of $W$ in $G$. Evidently, for any element $h \in G$ such that $h \notin \operatorname{Sep}_{(G, A)}(M, W)$ modules $W$ and $W h$ - are separated in $M$.

$k A$-modules $W$ and $V$ are said to be similar if their injective hulls $[W]$ and $[V]$ are isomorphic. The modules $W$ and $V$ are similar if and only if they 
have isomorphic essential submodules. By [3, Lemma 3.2], the stabilizer $S t a b_{G} W=\{g \in G \mid W g$ and $W$ are similar $\}$ of $W$ in $G$ is a subgroup of $G$.

We say that a submodule $X \leqslant M$ is solid in $M$ if $X$ is uniform and $M$ do not have submodules which are isomorphic to a proper section of $X$. It is easy to note that $\operatorname{Stab}_{G} W \leqslant \operatorname{Sep}_{(G, A)}(M, W)$, moreover, if the submodule $W$ is solid in $M$ then $\operatorname{Stab}_{G} W=\operatorname{Sep}_{(G, A)}(M, W)$.

Proposition 5.1. Let $N$ be a nilpotent normal non-abelian minimax torsionfree subgroup of a solvable-by-finite group $G$ such that $r_{0}(G)<\infty$. Let $K$ be an isolated $G$-invariant subgroup of $N$ such that the quotient group $N / K$ is abelian. Let $k$ be a field such that chark $=0$. Suppose that there is an uniform $k N$-torsion $k N$-module $W$ such that $S t a b_{G} W=G$ and for any proper isolated $G$-invariant subgroup $X$ of $N$ such that $K \leqslant X$ the module $W$ is $k X$-torsion-free. Then $\operatorname{Soc}_{G} N / K=N / K$.

Proof. Put $\operatorname{Soc}_{G} N / K=S / K$. Suppose that $\operatorname{Soc}_{G} N / K \neq N / K$ then it follows from Lemma 2.3(i) that $S$ is a proper isolated $G$-invariant subgroup of $N$ such that $K \leqslant S$ and hence the module $W$ is $k S$-torsion-free.

Let $L$ be a dense subgroup of $N$ such that $K \leqslant L$ and the quotient group $L / K$ is free abelian. As the subgroup $S$ is isolated in $N$, we see that $T=L \cap S$ is isolated in $L$ and hence we can choose a full system $\chi=\left\{\left\langle K,\left\{x_{j} \mid j \in J\right\}\right\rangle \mid J \subseteq\{1, \ldots, n\}\right\}$ of subgroups of $L$ over $\mathrm{K}$ such that $T \in \chi$. As the module $W$ is $k S$-torsion-free, the module $W$ is also $k T$ torsion-free.

By Theorem 4.1, there are a cyclic $k N$-submodule $0 \neq V \leqslant W$, a $G$-large ideal $I$ of $k K$ and a central $G$-invariant subgroup $A$ of finite index in $N / I^{\dagger}$ such that for any element $0 \neq b \in V$ we have:

(i) $X \in \chi(b k L)$ if and only if the quotient module $b k L / b k L I$ is not $k A_{X^{-}}$ torsion, where $A_{X}=A \cap X / I^{\dagger}$ and $A$ is central subgroup of finite index in $N / I^{\dagger}$;

(ii) for any dense subgroup $M \leqslant N$ with $K \leqslant M$ and finitely generated quotient group $M / K$ the finite set $M_{k A}(V / V I)=\left[\mu_{k A_{M}}(b k M / b k M I)\right]_{k A}$ depends only on the ideal $I$ and the subgroup $A$;

(iii) for any $g \in G$ we have $M_{k A}(V g / V g I)=M_{k A}(V / V I)^{g}=\left\{[P]^{g}=\right.$ $\left.\left[P^{g}\right] \mid[P] \in M_{k A}(V / V I)\right\}$. 
Since the module $W$ is $k T$-torsion-free, we have $T \in \chi(b k L)$ for any element $0 \neq b \in V$. As the module $W$ is uniform, we can conclude that $[V]=[W]$ and $[V g]=[W g]$ for any $g \in G$ and hence $[V g] \cong[V]$. It implies that $\operatorname{Stab}_{G} V=G$. Then for any $g \in G$ there are non-zero elements $b, c \in V$ such that $b k N \cong c k N g=c g k N$. Put $M=L \cap L^{-g}$ then it follows from (ii) that $M_{k A}(V / V I)=\left[\mu_{k A_{M}}(b k M / b k M I)\right]_{k A}=\left[\mu_{k A_{M}}(b g k M / b g k M I)\right]_{k A}=$ $M_{k A}(V g / V g I)$. Therefore, as by (iii) $M_{k A}(V g / V g I)=M_{k A}(V / V I)^{g}$, we see that $M_{k A}(V / V I)=M_{k A}(V / V I)^{g}=\left\{[P]^{g}=\left[P^{g}\right] \mid[P] \in M_{k A}(V / V I)\right\}$ and hence the group $G$ acts on the finite set $M_{k A}(V / V I)$. Then $\left|G: S_{G}([P])\right|<\infty$ for any $[P] \in M_{k A}(V / V I)$ and it easily implies that there is a subgroup $H$ of finite index in $G$ such that $[P]^{g}=\left[P^{g}\right]=[P]$ for any $[P] \in M_{k A}(V / V I)$ and any $g \in H$.

By Corollary 4.1, we have $M_{k A}(V / V I)=\left[\mu_{k A_{L}}(b k L / b k L I)\right]_{k A}$. Suppose that $M_{k A}(V / V I)=\left\{\left[P_{1}\right], \ldots,\left[P_{n}\right]\right\}$ then $B=A_{L}$ is a finitely generated dense subgroup of $A$ such that $\mu_{k B}\left(A n n_{k B}(b k L / b k L I)\right)=\left\{P_{i} \cap k B \mid 1 \leqslant i \leqslant n\right\}$. Put $C=A \cap S / I^{\dagger}$, as $S o c_{G} N / K=S / K$, we see that $C=S o c_{G} A$. Then, by Lemma 5.2, $A n n_{k B}(b k L / b k L I) \cap k(C \cap B) \neq 0$ and hence $A n n_{k B}(b k L / b k L I) \cap$ $k C \neq 0$. If $A_{T}=A \cap T / I^{\dagger}$, where $T=L \cap S$, then $A_{T}$ is a finitely generated dense subgroup of $C=A \cap S / I^{\dagger}$ and hence, as $A n n_{k B}(b k L / b k L I) \cap k C \neq 0$, it follows from [18, Lemma 2.2.3(ii)] that $A n n_{k B}(b k L / b k L I) \cap k A_{T} \neq 0$. It implies that the module $b k L / b k L I$ is $k A_{T}$-torsion. But it contradicts (i) because $T \in \chi(b k L)$. Thus, the obtained contradiction shows that $\operatorname{Soc}_{G} N / K=$ $N / K$.

Let $R$ be a ring and let $M$ be an $R$-module then $K_{R}(M)$ denotes the Krull dimension of $M$. The module $M$ is said to be $\rho$-critical if $K_{R}(M)=\rho$ and $K_{R}(M / U)<K_{R}(M)=\rho$ for any non-zero submodule $U \leqslant M$.

Let $N$ be a nilpotent group such that $r_{0}(N)<\infty$, let $k$ be a field and let $M$ be an $k N$-module. Let $S$ be a finitely generated subring of $k$. Let $0 \neq a \in M$ and let $H$ be a proper isolated normal subgroup of $N$. We say that $(a, H)$ is an important pair for the $S N$-module $M$ if there is a finitely generated dense subgroup $A \leqslant N$ such that:

(i) the module $a S A$ is $\rho$-critical and $K_{S X}(a S X) \leqslant K_{S X}(x S X)$ for any element $0 \neq x \in M$ and any finitely generated dense subgroup $X \leqslant N$;

(ii) $a S A=a S B \otimes_{S B} S A$, where $B=A \cap H$;

(iii) if $0 \neq b \in a S B$ and $V$ is a dense subgroup of $B$ then there is no isolated 
subgroup $D \leqslant V$ such that $b S V=b S D \otimes_{S D} S V$ and $i s_{N}(D)$ is a normal subgroup of $N$.

The module $M$ is said to be impervious if it has no important pairs for any finitely generated subring $S$ of $k$.

Proposition 5.2. Let $N$ be a nilpotent non-abelian minimax torsion-free normal subgroup of a soluble group $G$ such that $r_{0}(G)<\infty$. Let $K$ and $L$ be isolated $G$-invariant subgroups of $N$ such that $L \leqslant K, K$ contains next to the last member of the upper central series of $N$ and the quotient group $N / L$ is abelian. Let $k$ be a field such that chark $=0$. Let $W$ be a $k G$-module which is $k N$-torsion and for any proper isolated $G$-invariant subgroup $X$ of $N$ such that $L \leqslant X$ the module $W$ is $k X$-torsion-free. Suppose that $W$ is impervious $k N$-module. If $\operatorname{Sep}_{(G, Y)}(x k G, x k Y)=G$ for any element $0 \neq x \in W$ and any $G$-invariant subgroup $Y$ of $N$ then:

(i) $\operatorname{Soc}_{G} N / L=N / L$;

(ii) there is a dense $G$-invariant subgroup $D \leqslant N$ such that $K \leqslant D$ and the quotient group $D / K$ is polycyclic.

Proof. Evidently, there is no harm in assuming that the module $W=a k G$ is cyclic. Then, as the module $W=a k G$ is $k N$-torsion, there is a finitely generated subfield $f \leqslant k$ such $J=A n n_{f N}(a) \neq 0$ and hence, by Lemma 2.5, the module $W=a k G$ is $f N$-torsion. Since for any proper isolated $G$-subgroup $X$ of $N$ such that $K \leqslant X$ the module $W$ is $k X$-torsion-free, it is also $f X$-torsionfree. By [18, Lemma 3.1.2], $\operatorname{Sep}_{(G, k N)}(W, x k N) \leqslant \operatorname{Sep}_{(G, f N)}(W, x f N)$ and, as $\operatorname{Sep}_{(G, N)}(W, x k N)=G$, we may conclude that $\operatorname{Sep}_{(G, N)}(W, x f N)=G$. As the $k N$-module $x k N$ is impervious, it is easily to note that the $f N$-module $x f N$ is also impervious. Thus, there is no harm in assuming that the field $k$ is finitely generated. Then, by [18, Lemma 3.1.2], we can choose the element $x$ such that the $k N$-module $x k N$ is solid in $W$ and hence, the module $x k N$ is uniform and $\operatorname{Stab}_{G} x k N=\operatorname{Sep}_{(G, N)}(W, x k N)=G$.

(i) Since the module $x k N$ is uniform and $\operatorname{Stab}_{G} x k N=\operatorname{Sep}_{(G, N)}(W, x k N)=$ $G$, by Proposition 5.1, we have $\operatorname{Soc}_{G} N / K=N / K$.

(ii) It follows from (i) and Lemma 2.4(ii) that $G$ has a normal subgroup $H$ of finite index such that $\operatorname{Soc}_{G} N / K=N / K$ contains a dense $G$-invariant Noetherian $H$-polyplinth $Y / K$. As the subgroup $Y$ is dense in $N$ and the module $W$ is $k N$-torsion, it follows from [18, Lemma 2.2.3(ii)] that the module $W$ is $k Y$-torsion. Since $W$ is impervious as a $k N$-module, $W$ is impervious as a $k Y$-module. Then, as $\operatorname{Sep}_{(G, Y)}(x k G, x k Y)=G$, there is no harm 
in changing $N$ by $Y$, and we can assume that $A=N / K$ is a Noetherian $H$-polyplinth for some normal subgroup $H$ of finite index in $G$. After these changings, by [18, Lemma 3.1.2], we can choose the element $x$ such that the $k N$-module $x k N$ is solid in $W$ and hence, the module $x k N$ is uniform and $S t a b_{G} x k N=\operatorname{Sep}_{(G, N)}(W, x k N)=G$. By Lemma 2.4(iii), $\Delta_{G}(A)=D / K$ is a finitely generated isolated $G$-invariant subgroup of $A=N / K$ and, by Lemma $2.3(\mathrm{i}), \Delta_{G}\left(A / \Delta_{G}(A)\right)$ is trivial. Then, as the group $D / K$ is polycyclic, changing $K$ by $D$ we can assume that $\Delta_{G}(A)$ is trivial. Since $S t a b_{G} x k N=\operatorname{Sep}_{(G, N)}(W, x k N)=G$, we have $\operatorname{Stab}_{H} x k N=H$. Then it follows from [18, Lemma 3.3.4] that the quotient group $N / K$ is polycyclic.

Proposition 5.3. Let $N$ be a nilpotent non-abelian minimax torsion-free normal subgroup of a soluble group $G$ with $r_{0}(G)<\infty$. Let $k$ be a field such that chark $=0$. Let $W$ be a $k G$-module which is $k N$-torsion and for any proper isolated $G$-invariant subgroup $X$ of $N$ the module $W$ is $k X$-torsionfree. Suppose that $W$ is impervious as a $k N$-module and $\operatorname{Sep}_{(G, Y)}(x k G, x k Y)=$ $G$ for any element $0 \neq x \in W$ and any $G$-invariant subgroup $Y$ of $N$. Then for any finitely generated subgroup $H$ of $G$ the subgroup $N$ has an $H$-invariant polycyclic torsion-free non-abelian section.

Proof. Let $1=N_{m} \leqslant N_{m-1} \leqslant \ldots \leqslant N_{2} \leqslant N_{1} \leqslant N_{0}=N$ be the upper central series of the subgroup $N$. Put $K=N_{1}$ and $M=N_{2}$ then $N^{\prime} \leqslant K$. Let $L=i s_{N}\left(M N^{\prime}\right)$ then we have a series $M \leqslant L \leqslant K \leqslant N$ of $G$-invariant isolated subgroups of $N$ such that $N^{\prime} \leqslant L, M N^{\prime}$ is a dense subgroup of $L$ and $K / M$ is the centre of $N / M$. By Proposition 5.2, $\operatorname{Soc}_{G} N / L=N / L$ and there is a dense $G$-invariant subgroup $D \leqslant N$ such that $K \leqslant D$ and the quotient group $D / K$ is polycyclic. As $\operatorname{Soc}_{G} N / L=N / L$, we have $\operatorname{Soc}_{G} D / L=D / L$ and hence, by Lemma $2.4(\mathrm{iv})$, there is a $G$-invariant subgroup $P \leqslant D$ such that $L \leqslant P$, the quotient group $P / L$ is polycyclic, $P \cap K=L$ and $P K$ has finite index in $D$. Then, as $D / M$ is a nilpotent torsion-free group of nilpotency class two the assertion follows from Lemma 2.1.

\section{Primitive Representations of Finitely Gen- erated Linear Groups of Finite Rank}

Theorem 6.1. Let $G$ be an infinite finitely generated linear group of finite rank and let $k$ be a field of characteristic zero. If the group $G$ has a faithful primitive irreducible representation over the field $k$ then $|\Delta(G)|=\infty$. 
Proof. If the group $G$ is polycyclic-by-finite then the result follows from 6 , Theorem A]. So, we may assume that the group $G$ is not polycyclic-by-finite. Suppose that the group $G$ has a faithful primitive irreducible representation over the field $k$ and let $M$ be the module of this representation. By Lemma 2.2(ii), $G$ has a finite series $L \leqslant G_{0} \leqslant G$ of normal subgroups such that $\left|G / G_{0}\right|<\infty$, the quotient group $G_{0} / L$ is polycyclic and the subgroup $L$ is torsion-free nilpotent minimax which has no non-abelian torsion-free polycyclic $G$-invariant sections. It follows from [22, Theorem A] that $M$ contains a simple $k G_{0}$-module $M_{0}$. By [18, Theorem 4.2.5], the module $M_{0}$ is not $k L$-torsion-free and hence so is $M$. Then, as $r(G)<\infty$, there is an isolated $G$-invariant subgroup $N \leqslant L$ such that the module $M$ is not $k N$-torsion-free but for any proper isolated $G$-invariant subgroup $X \leqslant N$ the module $M$ is $k X$-torsion-free. It follows from Lemma 2.5 that the module $M$ is $k N$-torsion. As the module $M$ is irreducible and primitive, it follows from [18, Lemma 3.1.4(ii)] that $\operatorname{Sep}_{(G, Y)}(x k G, x k Y)=G$ for any element $0 \neq x \in M$ and any $G$-invariant subgroup $Y$ of $N$. If the $k N$-module $M$ is not impervious then it follows from [18, Proposition 3.2.3] that there are an element $0 \neq x \in M$ and a proper isolated subgroup $Y \leqslant N$ such that the module $M$ is not $k Y$-torsionfree and $\operatorname{Sep}_{(G, N)}(x k G, x k N) \leqslant N_{G}(Y)$. Since $\operatorname{Sep}_{(G, Y)}(x k G, x k Y)=G$, we see that $G=N_{G}(Y)$ but it is impossible because by the choice of $N$ the module $M$ is $k Y$-torsion-free for any proper isolated $G$-invariant subgroup $Y \leqslant N$. Thus, $M$ is impervious as a $k N$-module.

Suppose that $|\Delta(G)|<\infty$, as the subgroup $N$ is torsion-free it implies that $\Delta(G)=1$. If the subgroup $N$ is abelian then by [16, Theorem 4.2] there are an element $0 \neq x \in M$ and a subgroup $Y \leqslant N$ such that $x k G=(x k Y) \otimes_{k Y} k G$ and $r_{0}\left(Y / C_{Y}(x k Y)\right)<r_{0}(G)$. Since the module $M$ is irreducible, we have $M=x k G$ and, as the module $M$ is primitive, we see that $Y=G$. Therefore, $r_{0}\left(G / C_{G}(M)\right)<r_{0}(G)$ but it is impossible because $C_{G}(M)=1$.

So, the subgroup $N$ is non-abelian. Then, by Proposition 5.3, the subgroup $N$ has a torsion-free polycyclic non-abelian $G$-invariant section but it contradicts the choice of $N$. Thus, $|\Delta(G)|=\infty$.

\section{References}

[1] N. Bourbaki, Elements of Mathematics: Commutative Algebra, Chapters 1-7 (Springer, 1998). 
[2] C. J. B. Brookes, Ideals in group rings of soluble groups of finite rank, Math. Proc. Cambridge. Phil. Soc. 97 (1985) 27-49.

[3] C. J. B. Brookes and K. A. Brown, Primitive group rings and Noetherian rings of quotients, Trans. Amer. Math. Soc. 288 (1985) 605-623.

[4] C. J. B. Brookes, Modules over polycyclic groups, Proc. London Math. Soc. 57 (1988) 88-108.

[5] D. L. Harper, Primitive irreducible representations of nilpotent groups, Math. Proc. Camb. Phil. Soc. 82 (1977) 241-247.

[6] D. L. Harper, Primitivity in representations of polycyclic groups, Math. Proc. Camb. Phil. Soc. 88 (1980) 15-31.

[7] M. I. Kargapolov and Yu.I. Merzljakov, Fundamentals of the Theory of Groups, Graduate Texts in Math. ( Springer, 1979).

[8] J. C. Lennox and D.J.S. Robinson, The Theory of Infinite Soluble Groups (Clarendon Press, 2004).

[9] D. S. Passman, Infinite Crossed Products (Academic Press, 1989).

[10] D. J. S. Robinson, A Course in the Theory of Groups (Springer, 1996).

[11] F. Szechtman, Groups having a faithful irreducible representation, $J$. Algebra 454 (2016) 292-307.

[12] F. Szechtman and A. Tushev, Infinite groups admitting a faithful irreducible representation, J. Algebra Appl. 17 (2017) 1850005-1 - 18500057 .

[13] A. V. Tushev, Irreducible representations of locally polycyclic groups over an absolute field, Ukrainian Math. J. 42 (1990) 1233-1238.

[14] A. V. Tushev, On exact irreducible representations of locally normal groups, Ukrainian Mat. J. 45 (1993) 1900-1906.

[15] A. V. Tushev, On the primitivity of group algebras of certain classes of soluble groups of finite rank, Sb. Math. 186 (1995) 447-463. 
[16] A. V. Tushev, Spectra of conjugated ideals in group algebras of abelian groups of finite rank and control theorems, Glasgow Math. J. 38 (1996) 309-320.

[17] A. V. Tushev, Induced modules over group algebras of metabelian groups of finite rank, Comm. Algebra 27 (1999) 5921-5938.

[18] A. V. Tushev, On the primitive representations of soluble groups of finite rank, Sb. Math. 191 (2000) 117-159.

[19] A. V. Tushev, On Primitive Representations of Minimax Nilpotent Groups, Mathematical Notes 72 (2002) 117-128.

[20] A. V. Tushev, On the irreducible representations of soluble groups of finite rank, Asian-European J. Math. 5 (2012) 1250061-1-1250061-12.

[21] A. V. Tushev, On certain methods of studying ideals in group rings of abelian groups of finite rank, Asian-European J. Math. 7 (2014) 14500651- 1450065-7

[22] J. S. Wilson, Some properties of groups inherited by normal subgroups of finite index, Math. Z. 144 (1970) 19-21.

[23] J. S. Wilson, Soluble products of minimax groups, and nearly surjective derivations, J. Pure Appl. Algebra 53 (1988) 297-331.

[24] B. A. F. Wehrfritz, Infinite linear groups (Springer-Verlag, 1973).

[25] B. A. F. Wehrfritz, Group and Ring Theoretic Properties of Polycyclic Groups, Algebra and Applications, Vol. 10 (Springer, 2009).

[26] O. Zarisski and P. Samuel, Commutative Algebra, Vol. 1 (Van Nostrand, 1958). 